\title{
A Difference Method for Boundary Value Problems of the Third Kind*
}

\author{
By \\ Tatsuo Nogr**
}

\section{Introduction}

Pure difference methods for elliptic boundary value problems with derivative boundary conditions are treated by Batschelet [1], Giese [2], Lebedev [3-8], Volkov [9-10] and Wigley [11], etc.

For the same problems a kind of difference methods, what is called "Finite-element-method", are also investigated by Demjanovic [12], Friedrichs and Keller [13], Oganesyan [14-15], Oganesyan and Rukovetz [16-17] and Zlamál [18-19], etc. In this method a reduced minimal problem from the original boundary value problem is solved approximately in a subspace spanned by a class of finite number of "element" functions and their translated functions. The resulting difference scheme approximates automatically the differential equation in the interior of the domain and the boundary condition at points near the boundary. In these works the estimate of error between the exact and approximate solutions is given either in order of mesh width or precisely in an explicit form.

On the other hand, as far as we know, there were few works about difference methods for hyperbolic and parabolic mixed initial and boundary value problems with derivative boundary conditions in a domain of any shape. From mathematical interest we can refer to Lions [20] and Chekhlov [21] whose method is called "penalty method", in which the

Received January 27, 1971.

Communicated by S. Matsuura.

* This paper is the main part of the author's dissertation.

** Department of Applied Mathematics and Physics, Faculty of Engineering, Kyoto University, Yoshida, Kyoto, Japan. 
problems with homogeneous mixed (Dirichlet and Neumann) boundary conditions are considered and are reduced to the problem of a differential equation with some extended coefficients over the region and with the homogeneous Dirichlet condition. But the rate of convergence is at most $O(\sqrt{h})$, which shows that this method is not fit for practical use. ( $h$ is mesh width.)

Here we propose a difference method with rate of convergence $O(h)$ for mixed initial and boundary value problems of wave equation and heat equation with the boundary condition of third kind (and also for boundary value problems of elliptic equations) in a fairly arbitrary region on the plane. Our difference scheme corresponds to an integral formula of the original differential problem and has natural structures. The proof of convergence relies on the so called energy method (cf. Ladyzhenskaya [22]).

By trivial modification our method will be easily applied to the 3dimensional case, to the equations with variable coefficients and to the problem with mixed boundary conditions.

\section{§1. A Mixed Problem of a Wave Equation under a Derivative Boundary Condition and Its Difference Approximation}

We consider the mixed problem of the wave equation in a cylindrical region $Q(T)=\Omega \times(0, T)$ in $R^{3}\left(\Omega\right.$ is a bounded domain in $\left.R^{2}\right)$

$$
\frac{\partial^{2} u}{\partial t^{2}}-\frac{\partial^{2} u}{\partial x^{2}}-\frac{\partial^{2} u}{\partial y^{2}}=f(t, x, y) \quad \text { in } Q(T)
$$

under the initial conditions

$$
\begin{aligned}
u(0, x, y) & =\varphi(x, y), \\
\frac{\partial u}{\partial t}(0, x, y) & =\psi(x, y),
\end{aligned}
$$

and the boundary condition on the lateral surface

$$
\frac{\partial u}{\partial n}-\delta u=g(t, x, y)
$$


Here $\frac{\partial}{\partial n}$ means the derivative along exterior normal to the boundary surface. $\delta$ is a constant. Under appropriate smoothness conditions of the boundary $\Gamma$ of $\Omega$ and the functions $f$ and $g$, as we know, a unique smooth solution exists [23]. Moreover we assume that at every point $P \in \Gamma$ there is a circle $S$ such that $\bar{S} \cap \bar{\Omega}=P$.

For the sake of the future treatment we transform the equation (1.1) in an integral form by integrating the equation over any $(t, x, y)$-region $\omega \times[t, t+\Delta t]$ and using the Green's formula:

$$
\begin{aligned}
& \iint_{\omega}\left[\frac{\partial u}{\partial t}\right.\left.(t+\Delta t, x, y)-\frac{\partial u}{\partial t}(t, x, y)\right] d x d y \\
&=\int_{t}^{t+\Delta t} d t \int_{\partial \omega} \frac{\partial u}{\partial n} d s+\int_{t}^{t+\Delta t} d t \iint_{\omega} f d x d y
\end{aligned}
$$

where $d s$ means the line element of the boundary of $\omega$.

Now we construct a net in $R^{2}$ whose nodes have coordinates of the form

$$
x=m h, y=n k \quad(m, n=0, \pm 1, \pm 2, \cdots)
$$

where $h$ and $k$ are distances between the two adjoining nodes in the $x$ direction and the $y$-direction respectively. Denote the set of all the nodes in $\Omega$ by $\Omega_{h}^{\prime}$.

We consider those nodes which adjoin to $\Omega_{h}^{\prime}$. We call a node adjoining to two nodes of $\Omega_{h}^{\prime}$ a boundary mesh point of the first kind and call a node adjoining to one node of $\Omega_{h}^{\prime}$ a boundary mesh point of the second kind. Now we draw the "broken" lines through half-integer points, but we erase a broken segment lying between a boundary mesh point of the second kind and the corresponding node of $\Omega_{h}^{\prime}$.

Here we supposed that $h$ and $k$ are so small that no nodes outside of $\Omega$ have three or more neighbouring nodes of $\Omega_{h}^{\prime}$. (It is possible under our assumption about $\Gamma$.) Then if there appears a node being contained in a triangular mesh whose sides consist of two broken segments and a part of the boundary of $\Omega$, we count it in the class of boundary mesh points 
of the first kind, so that we need not consider original adjoining boundary mesh points of the second kind. We express the set of the left nodes of $\Omega_{h}^{\prime}$ by $\Omega_{h}$, which we call the set of interior mesh points.

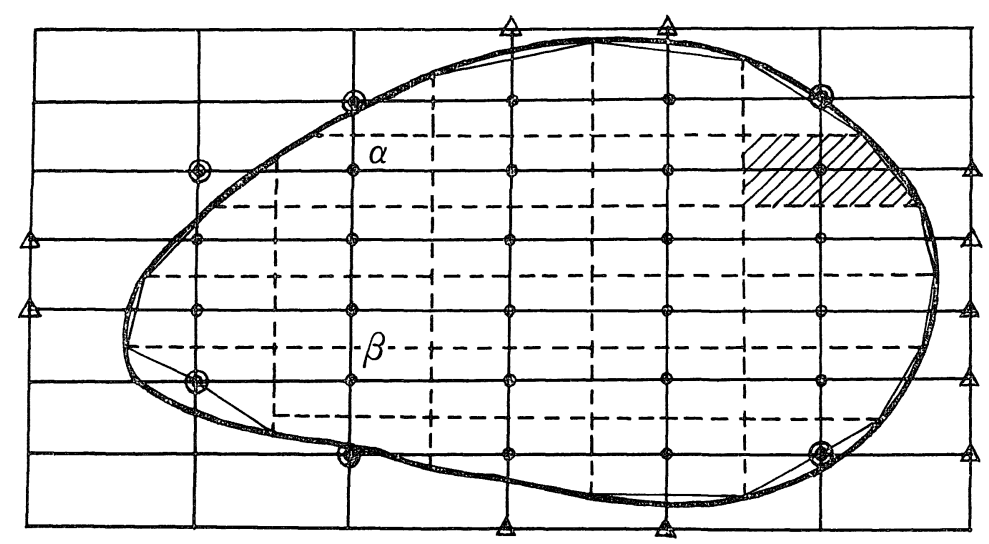

(2) The interior mesh point

() The boundary mesh point of first kind

$\triangle$ The boundary mesh point of second kind

Fig.-1 The concerned mesh points

And we express the set of all the boundary mesh points by $\Gamma_{h}$. Finally we draw line segments connecting the two neighbouring intersecting points of the "broken" line and the boundary, and then we have the polygonal region and express it by the same notation $\Omega_{h}$. The polygon $\Omega_{h}$ consists of some triangle, quadrilateral and pentagonal meshes having a boundary of broken lines or a side of the polygon.

In order to construct a difference scheme, we apply the integral formula (1.4) over each quadrilateral or pentagonal mesh and approximate each term by a corresponding difference quotient as follows; for example, over the hatched mesh in Fig. 1 we have a formula (after dividing by $\Delta t$ )

$$
S_{h} u_{t \bar{t}}=\left(\bar{g}+\delta\{u\}_{x+h}\right) \triangle \Gamma-a_{-h} u_{\bar{x}}+a_{+k} u_{y}-a_{-k} u_{\bar{y}}+S_{h} \bar{f},{ }^{1)}
$$

where $\triangle \Gamma, a_{-h}, a_{+k}$ and $a_{-k}$ are the length of the right, left, upper and lower side respectively, and $S_{h}$ means the area of the mesh. For the forward and

1) \{\}$_{x+h}$ means that the quantity in the bracket in calculated at the boundary mesh point of second kind, while other terms are calculated at the concerned node, 
backward difference quotients we employed the following notations;

$$
\begin{aligned}
& u_{t}=\frac{u(t+\Delta t, x, y)-u(t, x, y)}{\Delta t}, u_{\bar{t}}=\frac{u(t, x, y)-u(t-\Delta t, x, y)}{\Delta t}, \\
& u_{x}=\frac{u(t, x+h, y)-u(t, x, y)}{h}, u_{\bar{x}}=\frac{u(t, x, y)-(t, x-h, y)}{h}, \\
& u_{y}=\frac{u(t, x, y+k)-u(t, x, y)}{k}, u_{\bar{y}}=\frac{u(t, x, y)-u(t, x, y-k)}{k},
\end{aligned}
$$

and for difference quotients of second order, e.g.,

$$
\begin{aligned}
\left(u_{t}\right)_{\bar{t}} & =u_{t \bar{t}}=u_{\bar{t} t} \\
& \left.=\frac{1}{\Delta t^{2}}[u(t+\Delta t, x, y)-2 u(t, x, y)+u(t-\lrcorner t, x, y)\right] .
\end{aligned}
$$

$\bar{f}$ denotes the mean value of $f$ over the concerned mesh and $\bar{g}$ denotes the mean value of $g$ along the corresponding part of $\Gamma$. If we determine the value of $u$ at the boundary mesh point $\left(x_{0}, y_{0}\right)$ of the second kind adjacent to the concerned mesh by the formula

$$
\begin{aligned}
& a_{-h} u_{\bar{x}}=(\bar{g}+\delta u) \triangle \Gamma \quad \text { at }\left(x_{0}, y_{0}\right), \text { or equivalently } \\
& a_{+h} u_{x}=\left(\bar{g}+\delta\{u\}_{x+h}\right) \triangle \Gamma \text { at }\left(x_{0}-h, y_{0}\right), a_{-h}=a_{+h}=k,
\end{aligned}
$$

(which, we note, is only the replacement of notation and itself does not mean the formal approximation of the boundary condition), the equation (1.5) takes the form

$$
S_{h} u_{t \bar{t}}=a_{+h} u_{x}-a_{-h} u_{\bar{x}}+a_{+k} u_{y}-a_{-k} u_{\bar{y}}+S_{h} \bar{f}
$$

We can use the above difference equation (1.6) at any quadrilateral mesh if we take

$$
\begin{array}{ll}
a_{-h} u_{\bar{x}}=(\bar{g}+\delta u) \triangle \Gamma, \quad a_{-h}=k & \text { on a right boundary mesh point, } \\
a_{+h} u_{x}=-(\bar{g}+\delta u) \triangle \Gamma, a_{+h}=k & \text { on a left boundary mesh point, }
\end{array}
$$




$$
\begin{aligned}
& a_{-k} u_{\bar{y}}=(\bar{g}+\delta u) \Delta \Gamma, \quad a_{-k}=h \quad \text { on an upper boundary mesh point, } \\
& a_{+k} u_{y}=-(\bar{g}+\delta u) \triangle \Gamma, a_{+k}=h, \quad \text { on a lower boundary mesh point, }
\end{aligned}
$$

and $a_{+h}=a_{-h}=k, a_{+k}=a_{-k}=h$ on any interior mesh. ${ }^{2)}$

For a pentagonal mesh we have a formula

$$
S_{h} u_{t \bar{t}}=a_{+h} u_{x}-a_{-h} u_{\bar{x}}+a_{+k} u_{y}-a_{-k} u_{\bar{y}}+(\bar{g}+\delta u) \triangle \Gamma+S_{h} \bar{f}
$$

where $a_{+h}, a_{-h}, a_{+k}$ and $a_{-k}$ are the length of the right, left, upper and lower side respectively, and $\triangle \Gamma$ is that of the side of the polygon $\Omega_{h}$. By using the function $\delta_{h}$ which equals to unity on a pentagonal mesh and equals zero on a quadrilateral mesh, (1.6) and (1.8) can be written together in the form

$$
S_{h} u_{t \bar{t}}=a_{+h} u_{x}-a_{-h} u_{\bar{x}}+a_{+k} u_{y}-a_{-k} u_{\bar{y}}+\delta_{h}(\bar{g}+\delta u) \Delta \Gamma+S_{h} \bar{f}
$$

In order to determine the value of $u$ at each boundary mesh point of the first kind facing a triangular mesh we apply one of the following formulae;

$$
\begin{aligned}
& a_{-k} u_{\bar{x}}+a_{-k} u_{\bar{y}}=(\bar{g}+\delta u) \Delta \Gamma \quad \text { at a right upper mesh point, } \\
& a_{-h} u_{\bar{x}}-a_{+k} u_{y}=(\bar{g}+\delta u) \Delta \Gamma \quad \text { at a right lower mesh point, } \\
& -a_{+h} u_{x}+a_{-k} u_{\bar{y}}=(\bar{g}+\delta u) \triangle \Gamma \quad \text { at a left upper mesh point } \\
& \text { and } \quad-a_{+h} u_{x}-a_{+k} u_{y}=(\bar{g}+\delta u) \triangle \Gamma \quad \text { at a left lower mesh point. }
\end{aligned}
$$

At a boundary mesh point of the first kind facing a quadrilateral mesh we mush apply another formula, for example, at a left lower point of Fig. 1 we use the formula

$$
-a_{+h} u_{x}+a_{-h}\left\{u_{\bar{x}}\right\}_{y+k}-a_{+k} u_{y}=(\bar{g}+\delta u) \triangle \Gamma
$$

2) From the construction of our net it is known that $\frac{a_{-h}}{h} \frac{a_{+h}}{h} \frac{a_{-k}}{k}$ and $\frac{a_{+k}}{k}$ are uniformly apart from zero and then $\frac{a_{-h}}{h}-\delta \Delta \Gamma$ etc. are not zẹo for sufficiently small $h$. 
where \{\}$_{y+k}$ means that the quantity in the bracket is calculated at $(x, y+k)$, while other terms are calculated at $(x, y)$, and other notations are the same as above. At other boundary mesh points we can have analogous formulae. The formulae (1.10) and (1.11) approximate formally the boundary condition $\frac{\partial u}{\partial n}-\delta u=g$ with the error of order $O(h)+O(k)$, while (1.7) does not.

We also approximate the initial conditions (1.2) by the formulae

$$
\begin{aligned}
& u(0, x, y)=\varphi(x, y) \\
& u(\triangle t, x, y)=\varphi(x, y)+\triangle t \psi(x, y) .
\end{aligned}
$$

Then we have the values of $u$ on the planes $t=0$ and $t=\Delta t$ by (1.12), and we can dermine the values of $u$ on $t=2 \Delta t, 3 \triangle t, \ldots$ successively by using (1.9), (1.10) and (1.11), etc.

\section{§2. Convergence of the Scheme}

Now we will prove that the solution of our difference scheme converges to the solution of the original problem (1.1), (1.2) and (1.3) under appropriate conditions.

We can rewrite the difference equation (1.9) in the form

$$
\begin{gathered}
S_{h} u_{t \bar{t}}=\frac{h}{2}\left(a_{+h} u_{x}\right)_{\bar{x}}+\frac{h}{2}\left(a_{-h} u_{\bar{x}}\right)_{x}+\frac{k}{2}\left(a_{+k} u_{y}\right)_{\bar{y}}+\frac{k}{2}\left(a_{-k} u_{\bar{y}}\right)_{y}+ \\
+\delta_{h}(\bar{g}+\delta u) \triangle \Gamma+S_{h} \bar{f}
\end{gathered}
$$

by using the fact that $a_{-h}(x)=a_{+h}(x-h)$ and $a_{-k}(y)=a_{+k}(y-k)$. We multiply the last equation by $\left(u_{t}+u_{\bar{t}}\right)$ and transform it with the aid of the following formulae:

$$
\begin{aligned}
u_{t \bar{t}}\left(u_{t}+u_{\bar{t}}\right) & =\left(u_{\bar{t}}^{2}\right)_{t}, \\
\left(a_{+h} u_{x}\right)_{\bar{x}}\left(u_{t}+u_{\bar{t}}\right) & =\left[a_{+h} u_{x}\left(u_{t}+u_{\bar{t}}\right)\right]_{\bar{x}}-\left\{a_{+h} u_{x}\left(u_{t}+u_{\bar{t}}\right)_{x}\right\}_{x-h}, \\
\left(a_{-h} u_{\bar{x}}\right)_{x}\left(u_{t}+u_{\bar{t}}\right) & =\left[a_{-h} u_{\bar{x}}\left(u_{t}+u_{\bar{t}}\right)\right]_{x}-\left\{a_{-h} u_{\bar{x}}\left(u_{t}+u_{\bar{t}}\right)_{\bar{x}}\right\}_{x+h}, \text { etc., }
\end{aligned}
$$


where a curled bracket with a suffix have the same meaning as in (1.11). Then we have the equation

$$
\begin{aligned}
S_{h}\left(u_{\bar{t}}^{2}\right)_{t}= & \frac{h}{2}\left[a_{+h} u_{x}\left(u_{t}+u_{\bar{t}}\right)\right]_{\bar{x}}+\frac{h}{2}\left[a_{-h} u_{\bar{x}}\left(u_{t}+u_{\bar{t}}\right)\right]_{x} \\
& +\frac{k}{2}\left[a_{+k} u_{y}\left(u_{t}+u_{\bar{t}}\right)\right]_{\bar{y}}+\frac{k}{2}\left[a_{-k} u_{\bar{y}}\left(u_{t}+u_{\bar{t}}\right)\right]_{y} \\
& -\frac{h}{2}\left\{a_{+h} u_{x}\left(u_{t}+u_{\bar{t}}\right)_{x}\right\}_{x-h}-\frac{h}{2}\left\{a_{-h} u_{\bar{x}}\left(u_{t}+u_{\bar{t}}\right)_{\bar{x}}\right\}_{x+h} \\
& -\frac{k}{2}\left\{a_{+k} u_{y}\left(u_{t}+u_{\bar{t}}\right)_{y}\right\}_{y-k}-\frac{k}{2}\left\{a_{-k} u_{\bar{y}}\left(u_{t}+u_{\bar{t}}\right)_{\bar{y}}\right\}_{y+k} \\
& +\delta_{h}(\bar{g}+\delta u)\left(u_{t}+u_{\bar{t}}\right) \triangle \Gamma+S_{h} \bar{f}\left(u_{t}+u_{\bar{t}}\right) .
\end{aligned}
$$

We multiply the last equation by $\Delta t$ and sum over $\Omega_{h} \times[t=s \Delta t$; $s=1,2, \cdots, p-1]$ :

(2.2) $\left.\sum_{\Omega_{h}} S_{h} u \frac{2}{t}\right|^{p \Delta t} \underset{\Delta t}{\Delta}=$

$$
\begin{aligned}
=\sum_{s=1}^{p-1} \Delta & t\left[\frac{1}{2} \sum_{i=\sum_{j}(j)} a_{+h} u_{x}\left(u_{t}+u_{\bar{t}}\right)-\frac{1}{2} \sum_{i=i_{L_{j}(j)-1}} a_{+h} u_{x}\left(u_{t}+u_{\bar{t}}\right)\right. \\
& +\frac{1}{2} \sum_{i=i_{j}(j)+1} a_{-h} u_{\bar{x}}\left(u_{t}+u_{\bar{t}}\right)-\frac{1}{2} \sum_{i=i_{j}(j)} a_{-h} u_{\bar{x}}\left(u_{t}+u_{\bar{t}}\right) \\
& +\frac{1}{2} \sum_{j=j_{i} G(i)} a_{+k} u_{y}\left(u_{t}+u_{\bar{t}}\right)-\frac{1}{2} \sum_{j=j_{L_{i}(i)-1}} a_{+k} u_{y}\left(u_{t}+u_{\bar{t}}\right) \\
& +\frac{1}{2} \sum_{j=j_{G_{i}^{(i)+1}}} a_{-k} u_{\bar{y}}\left(u_{t}+u_{\bar{t}}\right)-\frac{1}{2} \sum_{j=j_{i}(i)} a_{-k} u_{\bar{y}}\left(u_{t}+u_{\bar{t}}\right) \\
& -\frac{1}{2} \sum_{\Omega_{h}} h\left\{a_{+h} u_{x}\left(u_{t}+u_{\bar{t}}\right)_{x}\right\}_{x-h}-\frac{1}{2} \sum_{\Omega_{h}} h\left\{a_{-h} u_{\bar{x}}\left(u_{t}+u_{\bar{t}}\right)_{\bar{x}}\right\}_{x+h} \\
& -\frac{1}{2} \sum_{\Omega_{h}} k\left\{a_{+k} u_{y}\left(u_{t}+u_{\bar{t}}\right)_{y}\right\}_{y-k}-\frac{1}{2} \sum_{\Omega_{h}} k\left\{a_{-k} u_{\bar{y}}\left(u_{t}+u_{\bar{t}}\right)_{\bar{h}}\right\}_{y+k}+
\end{aligned}
$$




$$
\left.+\sum_{\Gamma_{h}^{\prime}}(g+\delta u)\left(u_{t}+u_{\bar{t}}\right) \triangle \Gamma+\sum_{\Omega_{h}} S_{h} \bar{f}\left(u_{t}+u_{\bar{t}}\right)\right]
$$

where $i_{G}(j), i_{L}(j)-x$-coordinate numbers of right and left ends of each row segment ${ }^{3)}$ of mesh points on $y=j k$ in $\Omega_{h}$, and $j_{G}(i), j_{L}(i)-y$-coordinate numbers of upper and lower ends of each column segment of mesh points on $x=i h$ in $\Omega_{h}$. $\sum_{\Gamma_{h}^{\prime}}$ means $\sum_{\Gamma_{h}} \delta_{h}$. Here we note the following relations:

$$
\begin{aligned}
&\left.a_{+h} u_{x}\left(u_{t}+u_{\bar{t}}\right)\right|_{i_{G}}=\left.a_{-h} u_{\bar{x}}\left(u_{t}+u_{\bar{t}}\right)\right|_{i_{G}+1}-\left.h a_{+h} u_{x}\left(u_{t}+u_{\bar{t}}\right)_{x}\right|_{i_{G}}, \\
&\left.a_{-h} u_{\bar{x}}\left(u_{t}+u_{\bar{t}}\right)\right|_{i_{L}}=\left.a_{+h} u_{x}\left(u_{t}+u_{\bar{t}}\right)\right|_{i_{L}-1}+\left.h a_{-h} u_{x}\left(u_{t}+u_{\bar{t}}\right) \bar{x}\right|_{i_{L}}, \\
&\left.a_{+k} u_{y}\left(u_{t}+u_{\bar{t}}\right)\right|_{j_{G}}=\left.a_{-k} u_{\bar{y}}\left(u_{t}+u_{\bar{t}}\right)\right|_{j_{G}+1}-\left.k a_{+k} u_{y}\left(u_{t}+u_{\bar{t}}\right)_{y}\right|_{j_{G}}, \\
&\left.a_{-k} u_{\bar{y}}\left(u_{t}+u_{\bar{t}}\right)\right|_{j_{L}}=\left.a_{+k} u_{y}\left(u_{t}+u_{\bar{t}}\right)\right|_{j_{L}-1}+\left.k a_{-k} u_{y}\left(u_{t}+u_{\bar{t}}\right)_{\bar{y}}\right|_{j_{L}}, \\
& \sum_{\Omega_{h}} h\left\{a_{+h} u_{x}\left(u_{t}+u_{\bar{t}}\right)_{x}\right\}_{x-h}+\sum_{\Omega_{h}} h\left\{a_{-h} u_{\bar{x}}\left(u_{t}+u_{\bar{t}}\right)_{\bar{x}}\right\}_{x+h} \\
&=\sum_{\Omega_{h}} h a_{+h} u_{x}\left(u_{t}+u_{\bar{t}}\right)_{x}+\sum_{\Omega_{h}} h a_{-h} u_{\bar{x}}\left(u_{t}+u_{\bar{t}}\right)_{\bar{x}}
\end{aligned}
$$

and

$$
\begin{aligned}
\sum_{\Omega_{h}} k\left\{a_{+k} u_{y}\left(u_{t}+u_{\bar{t}}\right)_{y}\right\}_{y-k}+\sum_{\Omega_{h}} k\left\{a_{-k} u_{\bar{y}}\left(u_{t}+u_{\bar{t}}\right)_{\bar{t}}\right\}_{y+k} \\
=\sum_{\Omega_{h}} k a_{+k} u_{y}\left(u_{t}+u_{\bar{t}}\right)_{y}+\sum_{\Omega_{h}} k a_{-k} u_{\bar{y}}\left(u_{t}+u_{\bar{t}}\right)_{\bar{y}}
\end{aligned}
$$

Applying these formulae to the right side of (2.2), we have

$$
\begin{aligned}
& \left.\sum_{\Omega_{h}} S_{h} u \frac{2}{t}\right|_{\Delta t} ^{p \Delta t}= \\
& =\sum_{s=1}^{p-1} \Delta t\left[\sum_{i=i_{G_{j}(j)+1}} a_{-h} u_{\bar{x}}\left(u_{t}+u_{\bar{t}}\right)-\sum_{i=i_{L^{\prime}}(j)-1} a_{+h} u_{x}\left(u_{t}+u_{\bar{t}}\right)+\right. \\
& +\sum_{j=j_{i}(i)+1} a_{-k} u_{\bar{y}}\left(u_{t}+u_{\bar{t}}\right)-\sum_{j=j_{L_{i}(i)-1}} a_{+k} u_{y}\left(u_{t}+u_{\bar{t}}\right)+
\end{aligned}
$$

3) If $\Omega$ is not convex, some mesh lines $y=j k$ may be divided into several segments by the boundary. 


$$
\begin{aligned}
& -\frac{1}{2}\left(\sum_{\left.i G_{j}^{\prime} j\right)}+\sum_{\Omega_{h}}\right) h a_{+h} u_{x}\left(u_{t}+u_{\bar{t}}\right)_{x}-\frac{1}{2}\left(\sum_{i_{L_{j}(j)}}+\sum_{\Omega_{h}}\right) h a_{-h} u_{\bar{x}}\left(u_{t}+u_{\bar{t}}\right)_{\bar{x}} \\
& -\frac{1}{2}\left(\sum_{\substack{G_{i}(i) \\
a^{\prime}}}+\sum_{\Omega_{h}}\right) k a_{+k} u_{y}\left(u_{t}+u_{\bar{t}}\right)_{y}-\frac{1}{2}\left(\sum_{j_{L_{i}(i)}}+\sum_{h}\right) k a_{-k} u_{\bar{y}}\left(u_{t}+u_{\bar{t}}\right)_{\bar{y}} \\
& \left.+\sum_{\Gamma_{h}^{\prime}}(\bar{g}+\delta u)\left(u_{t}+u_{\bar{t}}\right) \Delta \Gamma+\sum_{\Omega_{h}} S_{h} \bar{f}\left(u_{t}+u_{\bar{t}}\right)\right] .
\end{aligned}
$$

Applying the boundary conditions (1.7), (1.10) and (1.11), we have

$$
\begin{gathered}
\left.\sum_{\Omega_{h}} S_{h} u \frac{2}{t}\right|_{\Delta \Delta} ^{p \Delta t}= \\
=-\frac{1}{2} \sum_{s=1}^{p-1} \Delta t\left[\left(\sum_{i_{G_{j}^{\prime}(j)}}+\sum_{\Omega_{h}}\right) h a_{+h} u_{x}\left(u_{t}+u_{\bar{t}}\right)_{x}+\left(\sum_{i_{L_{j}^{\prime}}(j)}+\sum_{\Omega_{h}}\right) h a_{-h} u_{\bar{x}}\left(u_{t}+u_{\bar{t}}\right)_{\bar{x}}+\right. \\
\left.+\left(\sum_{j G_{j}^{\prime}(i)}+\sum_{\Omega_{h}}\right) k a_{+k} u_{y}\left(u_{t}+u_{\bar{t}}\right)_{y}+\left(\sum_{j_{i}^{\prime}(i)}+\sum_{\Omega_{h}}\right) k a_{-k} u_{\bar{y}}\left(u_{t}+u_{\bar{t}}\right)_{\bar{y}}\right] \\
+\sum_{s=1}^{p-1} \Delta t\left[\sum_{\Gamma_{h}} \Delta \Gamma(\bar{g}+\delta u)\left(u_{t}+u_{\bar{t}}\right)+\sum_{\Gamma_{h}^{\prime}} \Delta \Gamma(\bar{g}+\delta u)\left(u_{t}+u_{\bar{t}}\right)+\right. \\
\left.+\sum_{\Omega_{h}} S_{h} \bar{f}\left(u_{t}+u_{\bar{t}}\right)\right] .
\end{gathered}
$$

We transform each term of the right side as follows:

$$
\begin{aligned}
& \sum_{s=1}^{p-1} \Delta t u_{x}\left(u_{t}+u_{\bar{t}}\right)_{x}=\sum_{s=0}^{p-1} \Delta t\left[u_{x} u_{x t}+\left\{u_{x} u_{x \bar{t}}\right\}_{(s+1) \Delta t}\right]-\left.\Delta t u_{x} u_{x t}\right|_{0} \\
& -\left.\triangle t u_{x} u_{x \bar{t}}\right|_{p \triangle t} \\
& =\left.u_{x}^{2}\right|_{0} ^{p \Delta t}-\left.\Delta t u_{x} u_{x \bar{t}}\right|_{p \Delta t}-\left.\Delta t u_{x} u_{x t}\right|_{0} \\
& \sum_{s=1}^{p-1} \Delta t u_{\bar{x}}\left(u_{t}+u_{\bar{x}}\right)_{\bar{t}}=\left.u_{\bar{x}}^{2}\right|_{0} ^{p \Delta t}-\left.\Delta t u_{\bar{x}} u_{\bar{x} \bar{t}}\right|_{p \Delta t}-\left.\Delta t u_{\bar{x}} u_{\bar{x} t}\right|_{0}, \\
& \sum_{s=1}^{p-1} \Delta t u_{y}\left(u_{t}+u_{\bar{t}}\right)_{y}=\left.u_{y}^{2}\right|_{0} ^{p \Delta t}-\left.\Delta t u_{y} u_{y \bar{t}}\right|_{p \Delta t}-\left.\Delta t u_{y} u_{y t}\right|_{0}, \\
& \sum_{s=1}^{p-1} \Delta t u_{\bar{y}}\left(u_{t}+u_{\bar{t}}\right)_{\bar{y}}=\left.u_{\bar{y}}^{2}\right|_{0} ^{p \Delta t}-\left.\Delta t u_{\bar{y}} u_{\bar{y} \bar{t}}\right|_{p \Delta t}-\left.\Delta t u_{\bar{y}} u_{\bar{y} t}\right|_{0}, \\
& \sum_{s=1}^{p-1} \Delta t \bar{g}\left(u_{t}+u_{\bar{t}}\right)=\sum_{s=1}^{p-1} \Delta t\left[(u \bar{g})_{t}-\left\{u \bar{g}_{\bar{t}}\right\}_{(s+1) \Delta t}+(u \bar{g})_{\bar{t}}-\left\{u \bar{g}_{t}\right\}_{(s-1) \Delta t}\right] \\
& =\left.u \bar{g}\right|_{\Delta t} ^{p \Delta t}+\left.u \bar{g}\right|_{0} ^{(p-1) \Delta t}-\sum_{s=2}^{p} \Delta t u \bar{g}_{\bar{t}}-\sum_{s=0}^{p-2} \Delta t u \bar{g}_{t}
\end{aligned}
$$




$$
\begin{aligned}
= & (\bar{g}(p \Delta t)+\bar{g}((p-1) \Delta t)) u(p \Delta t) \\
& -\Delta t \bar{g}((p-1) \Delta t) u_{t}((p-1) \triangle t) \\
& -\sum_{s=2}^{p} \Delta t u \bar{g}_{\bar{t}}-\sum_{s=0}^{p-2} \Delta t u \bar{g}_{t} \\
& -(\bar{g}(\triangle t)+\bar{g}(0)) u(0)-\triangle t \bar{g}(\triangle t) u \bar{t}(\triangle t)
\end{aligned}
$$

and

$$
\begin{aligned}
\sum_{s=1}^{p-1} \Delta t \delta u\left(u_{t}+u_{\bar{t}}\right)= & \sum_{s=0}^{p-1} \triangle t \delta\left(u^{2}\right)_{t}-\triangle t \delta u(0) u_{t}(0)-\triangle t \delta u(p \triangle t) u_{\bar{t}}(p \triangle t) \\
= & \delta u^{2}(p \triangle t)-\triangle t \delta u(p \triangle t) u_{\bar{t}}(p \triangle t) \\
& -\delta u^{2}(0)-\triangle t \delta u(0) u_{t}(0) .
\end{aligned}
$$

Thus we have

$$
\begin{aligned}
& \left.\sum_{\Omega_{h}} S_{h} u \frac{2}{t}\right|_{\triangle \Delta} ^{D_{\Delta} t} \\
& =-\frac{1}{2}\left[\left(\sum_{\substack{i_{G}^{(j j)} \\
j}}+\sum_{\Omega_{h}}\right) h a_{+h} u_{x}^{2}+\left(\sum_{i_{L_{j}^{(j)}}}+\sum_{\Omega_{h}}\right) h a_{-h} u \frac{2}{x}+\right. \\
& \left.+\left(\sum_{j G_{i}^{(i)}}+\sum_{\Omega_{h}}\right) k a_{+k} u_{y}^{2}+\left(\sum_{j L_{i}^{(i)}}+\sum_{\Omega_{h}}\right) k a_{-k} u \frac{2}{y}\right]\left.\right|_{0} ^{p \Delta t} \\
& +\frac{\triangle t}{2}\left[\left(\sum_{i_{\left.L_{j}^{\prime} j\right)}}+\sum_{\Omega_{h}}\right) h a_{+h} u_{x} u_{x \bar{t}}+\left(\sum_{i_{G_{j}(j)}}+\sum_{\Omega_{h}}\right) h a_{-h} u_{\bar{x}} u_{\bar{x} \bar{t}}+\right. \\
& \left.+\left(\sum_{j G_{i}^{(i)}}+\sum_{\Omega_{h}}\right) k a_{+k} u_{y} u_{y \bar{t}}+\left(\sum_{j_{L_{i}^{\prime}}(i)}+\sum_{\Omega_{h}}\right) k a_{-k} u_{\bar{y}} u_{\bar{y} \bar{t}}\right]_{p \triangle t}+ \\
& +\frac{\triangle t}{2}\left[\left(\sum_{\substack{i G_{j}^{(j)} \\
j}}+\sum_{\Omega_{h}}\right) h a_{+h} u_{x} u_{x t}+\left(\sum_{i_{L_{j}(j)}}+\sum_{\Omega_{h}} h a_{-h} u_{\bar{x}} u_{\bar{x} t}\right.\right. \\
& \left.+\left(\sum_{j G_{i}^{(i)}}+\sum_{\Omega_{h}}\right) k a_{+k} u_{y} u_{y t}+\left(\sum_{j_{L}^{(i)}}+\sum_{\Omega_{h}}\right) k a_{-k} u_{\bar{y}} u_{\bar{y} t}\right]_{0} \\
& +\left.\left(\sum_{\Gamma_{h}}+\sum_{\Gamma_{h}^{\prime}}\right) \triangle \Gamma \delta u^{2}\right|_{0} ^{p \Delta t}-\Delta t\left(\sum_{\Gamma_{h}}+\sum_{\Gamma_{h}^{\prime}}\right) \Delta \Gamma \delta u(p \Delta t) u_{\bar{t}}(p \Delta t) \\
& -\triangle t\left(\sum_{\Gamma_{h}}+\sum_{\Gamma_{h}^{\prime}}\right) \triangle \Gamma \delta u(0) u_{t}(0)
\end{aligned}
$$




$$
\begin{aligned}
& +\left(\sum_{\Gamma_{h}}+\sum_{\Gamma_{h}^{\prime}}\right) \Delta \Gamma[(\bar{g}(p \Delta t)+\bar{g}((p-1) \Delta t)) u(p \Delta t) \\
& \left.\quad-\Delta t \bar{g}((p-1) \Delta t) u_{t}((p-1) \Delta t)\right] \\
& -\left(\sum_{\Gamma_{h}}+\sum_{\Gamma_{h}^{\prime}}\right) \Delta \Gamma\left[(\bar{g}(\Delta t)+\bar{g}(0)) u(0)-\Delta t \bar{g}(\Delta t) u_{\bar{t}}(\Delta t)\right] \\
& -\sum_{s=2}^{p} \Delta t\left(\sum_{\Gamma_{h}}+\sum_{\Gamma_{h}^{\prime}}\right) \Delta \Gamma u \bar{g} \bar{t}-\sum_{s=0}^{p-2} \Delta t\left(\sum_{\Gamma_{h}}+\sum_{\Gamma_{h}^{\prime}}\right) u \bar{g}_{t} \\
& +\sum_{s=1}^{p-1} \Delta t \sum_{\Omega_{h}} S_{h} \bar{f}\left(u_{t}+u_{\bar{t}}\right) .
\end{aligned}
$$

Here we transform the second bracket of the right side as follows:

$$
\begin{aligned}
& \frac{\triangle t}{2}\left[\left(\sum_{i_{G_{j}^{(j)}}}+\sum_{\Omega_{h}}\right) h a_{+h} u_{x} u_{x \bar{t}}+\left(\sum_{i_{L_{j}^{(j)}}}+\sum_{\Omega_{h}}\right) h a_{-h} u_{\bar{x}} u_{\bar{x} \bar{t}}+\right. \\
& \left.+\left(\sum_{j G_{i}^{(i)}}+\sum_{\Omega_{h}}\right) k a_{+k} u_{y} u_{y \bar{t}}+\left(\sum_{j L_{i}^{(i)}}+\sum_{\Omega_{h}}\right) k a_{-k} u_{\bar{y}} u_{\bar{y} \bar{t}}\right] \\
& =\Delta t\left[\left(\sum_{\substack{i G_{j}(j) \\
j}}+\frac{1}{2} \sum_{\substack{i \neq i \in G(j) \\
j}}\right) a_{+h} u_{x}\left(u_{\bar{t}}(x+h)-u_{\bar{t}}(x)\right)\right. \\
& +\left(\sum_{\substack{i_{L_{j}(j)} \\
j}}+\frac{1}{2} \sum_{\substack{i \neq i_{L_{j}(j)} \\
j}}\right) a_{-h} u_{\bar{x}}\left(u_{\bar{t}}(x)-u_{\bar{t}}(x-h)\right) \\
& +\left(\sum_{\substack{\left.j_{G} \backslash i\right) \\
i}}+\frac{1}{2} \sum_{\substack{j \neq j_{G} G(i) \\
i}}\right) a_{+k} u_{y}\left(u_{\bar{t}}(y+k)-u_{\bar{t}}(y)\right) \\
& \left.+\left(\sum_{j_{L}(i)}+\frac{1}{2} \sum_{j \neq j_{i}(i)}\right) a_{-k} u_{\bar{y}}\left(u \bar{t}(y)-u_{\bar{t}}(y-k)\right)\right] \\
& =\Delta t\left[\left(\sum_{\Gamma_{h}}+\sum_{\Gamma_{h}^{\prime}}\right) \Delta \Gamma(\bar{g}+\delta u) u_{\bar{t}}-\right. \\
& -\sum_{\substack{G_{G}(j) \\
j}} a_{+h} u_{x} u_{\bar{t}}+\sum_{\substack{i_{L^{(j)}} \\
j_{j}}} a_{-h} u_{\bar{x}} u_{\bar{t}}-\sum_{j_{G_{i}(i)}} a_{+k} u_{y} u_{\bar{t}}+\sum_{j_{L_{i}}(i)} a_{-k} u_{\bar{y}} u_{\bar{t}}+ \\
& +\frac{1}{2} \sum_{\substack{i \neq i_{G}(j) \\
j}} a_{+h} u_{x}\left(u_{\bar{t}}(x+h)-u_{\bar{t}}(x)\right)+\frac{1}{2} \sum_{\substack{i \neq i_{L}(j) \\
j}} a_{-h} u_{\bar{x}}\left(u_{\bar{t}}(x)-\right. \\
& \left.-u_{\bar{t}}(x-h)\right)+\frac{1}{2} \sum_{j \neq j_{i}(i)} a_{+k} u_{y}\left(u_{\bar{t}}(y+k)-u_{\bar{t}}(y)\right)+
\end{aligned}
$$




$$
\left.+\frac{1}{2} \sum_{j \neq j_{i}(i)} a_{-k} u_{\bar{y}}\left(u_{\bar{t}}(y)-u_{\bar{t}}(y-k)\right)\right] .
$$

By the last relation and intial conditions (1.12), the equation (2.3) takes the form

$$
\begin{aligned}
& \text { (2.4) } \sum_{\Omega_{h}} S_{h} u \frac{2}{t}(p \triangle t)+\frac{1}{2}\left[\left(\sum_{\substack{i G_{j}^{(j)} \\
j}}+\sum_{\Omega_{h}}\right) h a_{+h} u_{x}^{2}+\left(\sum_{\substack{i \\
L_{j}^{(j)}}}+\sum_{\Omega_{h}}\right) h a_{-h} u \frac{2}{x}+\right. \\
& \left.+\left(\sum_{j G_{i}^{(i)}}+\sum_{\Omega_{h}}\right) k a_{+k} u_{y}^{2}+\left(\sum_{j L_{i}^{(i)}}+\sum_{\Omega_{h}}\right) k a_{-k} u \frac{2}{y}\right]_{p \Delta t} \\
& =\Delta t\left[-\sum_{i_{G_{j}(j)}} a_{+k} u_{x} u_{\bar{t}}+\sum_{i_{L_{j}^{(j)}}} a_{-h} u_{\bar{x}} u_{\bar{t}}-\sum_{j_{G_{i}^{(i)}}} a_{+k} u_{y} u_{\bar{t}}+\sum_{j_{L_{i}}(i)} a_{-k} u_{\bar{y}} u_{\bar{t}}\right. \\
& +\frac{1}{2} \sum_{\substack{i \neq i_{G}(j) \\
j}} a_{+h} u_{x}\left(u_{\bar{t}}(x+h)-u_{\bar{t}}(x)\right)+\frac{1}{2} \sum_{\substack{i \neq i_{L}(j) \\
j}} a_{-h} u_{\bar{x}}\left(u_{\bar{t}}(x)-u_{\bar{t}}(x-h)\right) \\
& +\frac{1}{2} \sum_{j \neq j_{G}(i)} a_{+k} u_{y}\left(u_{\bar{t}}(y+k)-u_{\bar{t}}(y)\right)+\frac{1}{2} \sum_{\substack{j \neq j_{G}(i) \\
i}} a_{-k} u_{\bar{y}}\left(u_{\bar{t}}(y)-\right. \\
& \left.\left.-u_{\bar{t}}(y-k)\right)\right] p \Delta t \\
& +\left(\sum_{\Gamma_{h}}+\sum_{\Gamma_{h}^{\prime}}\right) \Delta \Gamma\left[\delta u^{2}-\triangle t \delta u u_{\bar{t}}\right]_{p \Delta t} \\
& +\left(\sum_{\Gamma_{h}}+\sum_{\Gamma_{h}^{\prime}}\right) \triangle \Gamma[(\bar{g}(p \triangle t)+\bar{g}((p-1) \triangle t)) u(p \triangle t) \\
& \left.-\triangle t \bar{g}((p-1) \Delta t) u_{\bar{t}}(p \Delta t)\right] \\
& -\left(\sum_{\Gamma_{h}}+\sum_{\Gamma_{h}^{\prime}}\right) \Delta \Gamma\left[\sum_{s=0}^{p} \Delta t u \bar{g}_{\bar{t}}+\sum_{s=2}^{p-2} \Delta t u \bar{g}_{t}\right] \\
& +\sum_{s=1}^{p-1} \Delta t \sum_{\Omega_{h}} S_{h} \bar{f}\left(u_{t}+u_{\bar{t}}\right)+\sum_{\Omega_{h}} S_{h} \psi^{2} \\
& +\frac{1}{2}\left[\left(\sum_{i_{G_{j}}(j)}+\sum_{\Omega_{h}}\right) h a_{+h}\left(\varphi_{x}^{2}+\triangle t \varphi \psi_{x}\right)+\left(\sum_{i_{L_{j}}(j)}+\sum_{\Omega_{h}}\right) h a_{-h}\left(\varphi_{\bar{x}}^{2}+\triangle t \varphi \psi_{\bar{x}}\right)\right. \\
& \left.+\left(\sum_{j G_{i}^{(i)}}+\sum_{\Omega_{h}}\right) k a_{+k}\left(\varphi_{y}^{2}+\Delta t \varphi \psi_{y}\right)+\left(\sum_{j_{L}^{(i)}}+\sum_{\Omega_{h}}\right) k a_{-k}\left(\varphi \frac{2}{y}+\Delta t \varphi \psi_{y}^{-}\right)\right] \\
& -\left(\sum_{\Gamma_{h}}+\sum_{\Gamma_{h}^{\prime}}\right) \triangle \Gamma[(\bar{g}(\Delta t)+\bar{g}(0)) \varphi-\triangle t \bar{g}(\triangle t) \psi] .
\end{aligned}
$$


Each term in the right side of the last equation can be estimated as follows;

$$
\begin{aligned}
& \left|\Delta t\left[-\sum_{\substack{i G_{j}^{(j)} \\
j}} a_{+h} u_{x} u_{\bar{t}}+\sum_{i_{L_{j}^{\prime}(j)}} a_{-h} u_{\bar{x}} u_{\bar{t}}-\sum_{G_{i}^{(i)}} a_{+k} u_{y} u_{\bar{t}}+\sum_{j_{L_{i}^{(i)}}} a_{-k} u_{\bar{y}} u_{\bar{t}}\right]\right| \\
& \leqq \Delta t\left[\sum_{i G_{j}^{(j)}} a_{+h}\left(\frac{u^{\frac{2}{t}}}{8}+2 u_{x}^{2}\right)+\sum_{i_{L_{j}^{(j)}}} a_{-h}\left(\frac{u_{\bar{x}}^{2}}{8}+2 u_{\bar{x}}^{2}\right)+\sum_{j G_{i}^{(i)}} a_{+h}\left(\frac{u_{t}^{2}}{8}+2 u_{y}^{2}\right)\right. \\
& \left.+\sum_{j_{i}^{(i)}} a_{-k}\left(\frac{u_{\bar{t}}^{2}}{8}+2 u \frac{2}{y}\right)\right] \\
& \mid \frac{\triangle t}{2}\left[\sum_{\substack{i \neq i_{G}(j) \\
j}} a_{+h} u_{x}\left(u_{\bar{t}}(x+h)-u_{\bar{t}}(x)\right)+\sum_{\substack{i \neq i_{L}(j) \\
i}} a_{-h} u_{\bar{x}}\left(u_{\bar{t}}(x)-u_{\bar{t}}(x-h)\right)+\right. \\
& \left.+\sum_{j \neq j_{i} G(i)} a_{+k} u_{y}\left(u_{\bar{t}}(y+k)-u_{\bar{t}}(y)\right)+\sum_{j \neq j_{i}(i)} a_{-k} u_{\bar{y}}\left(u_{\bar{t}}(y)-u_{\bar{t}}(y-k)\right)\right] \mid \\
& \leqq \frac{\triangle t}{2}\left[\sum_{\substack{i \neq i_{j}(j) \\
j}} a_{+h}\left(\frac{u_{\bar{t}}(x+h)^{2}+u_{\bar{t}}(x)^{2}}{4}+2 u_{x}^{2}\right)+\right. \\
& +\sum_{\substack{i \neq i_{L}(j) \\
j}} a_{-h}\left(\frac{u_{\bar{t}}(x)^{2}+u_{\bar{t}}(x-h)^{2}}{4}+2 u_{\bar{x}} \frac{2}{x_{j \neq j_{i} G}(i)}+a_{+k}\left(\frac{u_{\bar{t}}(y+k)^{2}+u_{\bar{t}}(y)^{2}}{4}+2 u_{y}^{2}\right)\right. \\
& \left.+\sum_{j \neq j_{\bar{i}}(i)} a_{-k}\left(\frac{u_{\bar{t}}(y)^{2}+u_{\bar{t}}(y-k)^{2}}{4}+2 u \frac{2}{y}\right)\right] \\
& =\frac{\triangle t}{2}\left[\sum_{\substack{i=i_{G}(j) \\
j}} \frac{a_{+h}+a_{-h}}{4} u \frac{2}{t}+\sum_{\substack{i=i_{L}(j) \\
j}} \frac{a_{+h}+a_{-h}}{4} u_{\frac{2}{t}}+\sum_{\substack{i \neq i_{G}(j), i_{L}(j) \\
j}} \frac{a_{+h}+a_{-h}}{2} u \frac{2}{t}\right. \\
& +\sum_{j=j_{i}(i)} \frac{a_{+k}+a_{-k}}{4} u_{t}^{2}+\sum_{j=j_{L}(i)} \frac{a_{+k}+a_{-k}}{4} u_{i}^{2}+\sum_{j \neq j_{G}(i), j_{L}(i)} \frac{a_{+k}+a_{-k}}{2} u_{t}^{2} \\
& \left.+\sum_{\substack{j \neq i_{G}(j) \\
j}} 2 a_{+h} u_{x}^{2}+\sum_{\substack{j \neq i_{i}(j) \\
i}} 2 a_{-h} u \frac{2}{x}+\sum_{\substack{j \neq i_{G}(i) \\
t}} 2 a_{+k} u_{y}^{2}+\sum_{\substack{j \neq j_{L}(i) \\
i}} 2 a_{-k} u \frac{2}{y}\right], \\
& \left|\left(\sum_{\Gamma_{h}}+\sum_{\Gamma_{h}^{\prime}}\right) \triangle \Gamma(\bar{g}(p \triangle t)+\bar{g}((p-1) \Delta t)) u(p \triangle t)\right| \\
& \leqq\left(\sum_{\Gamma_{h}}+\sum_{\Gamma_{h}^{\prime}}\right) \Delta \Gamma\left[u(p \Delta t)^{2}+\frac{1}{2}\left(\bar{g}(p \Delta t)^{2}+\bar{g}((p-1) \Delta t)^{2}\right)\right] .
\end{aligned}
$$

And by using the boundary condition we have 


$$
\begin{aligned}
& \left|\Delta t\left(\sum_{\Gamma_{h}}+\sum_{\Gamma_{h}^{\prime}}\right) \Delta \Gamma \bar{g}((p-1) \Delta t) u_{\bar{t}}(p \Delta t)\right| \\
\leqq & \frac{1}{2}\left(\sum_{\Gamma_{h}}+\sum_{\Gamma_{h}^{\prime}}\right) \Delta \Gamma\left[\bar{g}((p-1) \Delta t)^{2}+\Delta t^{2} u_{\bar{t}}(p \Delta t)^{2}\right] \\
\leqq & C_{1} \Delta t \sum_{\Omega_{h}} S_{h} u_{\bar{t}}(p \Delta t)^{2}+\frac{1}{2}\left(\sum_{\Gamma_{h}}+\sum_{\Gamma_{h}^{\prime}}\right) \Delta \Gamma\left[\bar{g}((p-1) \Delta t)^{2}+C_{2} \sqrt{h k} \Delta t^{2} \bar{g}_{\bar{t}}^{2}\right],
\end{aligned}
$$

where $C_{1}$ and $C_{2}$ are constants independent of $\Delta t, h$ and $k$. Other terms in the right side of (2.4) are also estimated by Schwarz's inequality. Then we have the following inequality.

$$
\begin{aligned}
& \text { (2.5) } \sum_{\Omega_{h}} S_{h} u_{\bar{t}}(p \Delta t)^{2}+\frac{1}{2}\left[\left(\sum_{i G_{j}^{(j)}}+\sum_{\Omega_{h}}\right) h a_{+h} u_{x}^{2}+\left(\sum_{i L_{j}^{(j)}}+\sum_{\Omega_{h}}\right) h a_{-h} u_{\bar{x}}^{2}+\right. \\
& \left.+\left(\sum_{j G_{i}^{(i)}}+\sum_{\Omega_{h}}\right) k a_{+k} u_{y}^{2}+\left(\sum_{j_{i}(i)}+\sum_{\Omega_{h}}\right) k a_{-k} u_{\bar{y}}^{2}\right]_{p \Delta t} \leqq \\
& \leqq \triangle t\left[\sum_{\substack{i G_{j}(j) \\
j}}\left(\frac{a_{+h}}{4}+\frac{a_{-h}}{8}\right) u_{\bar{t}}^{2}+\sum_{\substack{i_{L^{\prime}}(j) \\
j}}\left(\frac{a_{-h}}{4}+\frac{a_{+h}}{8}\right) u_{t}^{2}+\sum_{j G_{i}^{(i)}}\left(\frac{a_{+k}}{4}+\frac{a_{-k}}{8}\right) u_{\bar{t}}^{2}\right. \\
& +\sum_{j_{L_{i}(i)}}\left(\frac{a_{-k}}{4}+\frac{a_{+k}}{8}\right) u_{\bar{t}}^{2}+\sum_{i \neq i_{G}(j), j_{j}(j)} \frac{a_{+h}+a_{-h}}{4} u_{\bar{t}}^{2}+\sum_{j \neq j_{G}(i), j_{i}(i)} \frac{a_{+k}+a_{-k}}{4} u_{\bar{t}}^{2}
\end{aligned}
$$

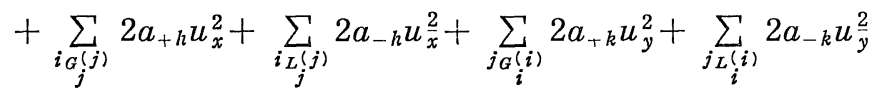

$$
\begin{aligned}
& \left.+\sum_{i \neq i_{j}(j)} a_{+h} u_{x}^{2}+\sum_{i \neq i_{j}(j)} a_{-h} u_{\bar{x}}^{2}+\sum_{j \neq j_{i} G(i)} a_{+k} u_{y}^{2}+\sum_{j \neq j_{L}(i)} a_{-k} u_{\bar{y}}^{2}\right]_{p \triangle t} \\
& +C_{1} \triangle t \sum_{\Omega_{h}} S_{h} u_{\bar{t}}(p \Delta t)^{2}+\sum_{s=1}^{p} \triangle t \sum_{\Omega_{h}} S_{h} u_{\bar{t}}^{2} \\
& +\left(\sum_{\Gamma_{h}}+\sum_{\Gamma_{h}^{\prime}}\right) \triangle \Gamma\left[\left(1+\frac{3}{2}|\delta|\right) u^{2}+\left(\frac{\triangle t^{2}}{2}|\delta|+\frac{\triangle t^{2}}{2}\right) u_{t}^{2}\right]_{p \Delta t} \\
& +\sum_{s=0}^{p-1} \triangle t\left(\sum_{\Gamma_{h}}+\sum_{\Gamma_{h}^{\prime}}\right) u(s \triangle t)^{2} \triangle \Gamma \\
& +\left(\sum_{\Gamma_{h}}+\sum_{\Gamma_{h}^{\prime}}\right) \Delta \Gamma\left[2 \bar{g}((p-1) \Delta t)^{2}+\Delta t^{2}\left(1+\frac{C_{2} \sqrt{h k}}{2}\right) g_{\bar{t}}(p \Delta t)^{2}\right. \\
& +\sum_{s=0}^{p-1} \Delta t\left(\sum_{\Gamma_{h}}+\sum_{\Gamma_{h}^{\prime}}\right) \Delta \Gamma \bar{g}_{t}^{2}+\sum_{s=1}^{p-1} \Delta t \sum_{\Omega_{h}} S_{h} \bar{f}^{2}+\sum_{\Omega_{h}} S_{h} \psi^{2}+
\end{aligned}
$$




$$
\begin{aligned}
& +\frac{1}{2}\left[\left(\sum_{i_{j}^{(j)}}+\sum_{\Omega_{h}}\right) h a_{+h}\left\{\left(1+\frac{\triangle t}{2}\right) \varphi_{x}^{2}+\frac{\Delta t}{2} \psi_{x}^{2}\right\}\right. \\
& +\left(\sum_{i_{L}(j)}+\sum_{\Omega_{h}}\right) h a_{-h}\left\{\left(1+\frac{\Delta t}{2}\right) \varphi_{\bar{x}}^{2}+\frac{\Delta t}{2} \psi_{\bar{x}}^{2}\right\} \\
& +\left(\sum_{j G_{i}^{(i)}}+\sum_{\Omega_{h}}\right) k a_{+k}\left\{\left(1+\frac{\triangle t}{2}\right) \varphi_{y}^{2}+\frac{\triangle t}{2} \psi_{y}^{2}\right\} \\
& \left.+\left(\sum_{j_{L}(i)}+\sum_{\Omega_{h}}\right) k a_{-k}\left\{\left(1+\frac{\Delta t}{2}\right) \varphi_{\frac{2}{y}}+\frac{\Delta t}{2} \psi_{\frac{2}{y}}^{2}\right\}\right] \\
& +\frac{1}{2}\left(\sum_{\Gamma_{h}}+\sum_{\Gamma_{h}^{\prime}}\right) \Delta \Gamma\left[\bar{g}(0)^{2}+(1+\Delta t) \bar{g}(\Delta t)^{2}+\varphi^{2}+\Delta t \psi^{2}\right] .
\end{aligned}
$$

Here we note two lemmas.

Lemma 1. For any $u$ defined on $\Omega_{h}+\Gamma_{h}$, we have the inequality

$$
\begin{aligned}
& \sum_{\Gamma_{h}} u^{2} \Delta \Gamma+\sum_{\Gamma_{h}^{\prime}} u^{2} \Delta \Gamma \\
\leqq & \varepsilon \sum_{\Omega_{h}}\left[h\left(a_{+h} u_{x}^{2}+a_{-h} u_{\bar{x}}^{2}\right)+k\left(a_{+k} u_{y}^{2}+a_{-k} u_{y}^{2}\right)\right]+C(\varepsilon) \sum_{\Omega_{h}} S_{h} u^{2}
\end{aligned}
$$

where $\varepsilon$ is an arbitrarily small constant. The constant $C(\varepsilon)$ depends on $\varepsilon$ and becomes larger as $\varepsilon$ becomes smaller.

Proof of Lemma 1. We first note that for any $v$

$$
\begin{gathered}
\text { (2.6) } \sum_{\substack{i_{G}(j)+1 \\
j}} a_{+h}(x-h) v-\sum_{\substack{i_{L}(j)-1 \\
j}} a_{+h}(x) v=\sum_{\Omega_{h}+\left\{i_{L^{\prime}}(j)-1\right\}} h a_{+h} v_{x} \\
+\sum_{\Omega_{h}}\left(a_{+h}(x)-a_{+h}(x-h)\right) v
\end{gathered}
$$

where $\sum_{\Omega_{h}+\left\{i_{L}(j)-1\right\}}$ means the summation over all the mesh points of $\Omega_{h}$ and all the boundary mesh points having the coordinate numbers $i_{L}(j)-1$. In fact,

$$
\begin{gathered}
\sum_{\Omega_{h}+\left\{i_{L}(j)-1\right\}} h a_{+h} v_{x} \\
=\sum_{j}\left[a_{+h}\left(i_{G}\right)\left(v\left(i_{G}+1\right)-v\left(i_{G}\right)\right)+a_{+h}\left(i_{G}-1\right)\left(v\left(i_{G}\right)-v\left(i_{G}-1\right)\right)+\right. \\
\left.\quad+\cdots+a_{+h}\left(i_{L}\right)\left(v\left(i_{L}+1\right)-v\left(i_{L}\right)\right)+a_{+h}\left(i_{L}-1\right)\left(v\left(i_{L}\right)-v\left(i_{L}-1\right)\right)\right]
\end{gathered}
$$




$$
\begin{aligned}
=\sum_{j} a_{+h}\left(i_{G}\right) v\left(i_{G}+1\right)-\sum_{j} a_{+h}\left(i_{L}-1\right) v\left(i_{L}-1\right) \\
\quad-\sum_{j}\left[\left(a_{+h}\left(i_{G}\right)-a_{+h}\left(i_{G}-1\right)\right) v\left(i_{G}\right)+\left(a_{+h}\left(i_{G}-1\right)-a_{+h}\left(i_{G}-2\right)\right) v\left(i_{G}-1\right)+\right. \\
\left.\quad+\cdots+\left(a_{+h}\left(i_{L}\right)-a_{+h}\left(i_{L}-1\right)\right) v\left(i_{L}\right)\right] \\
\quad+\cdots \sum_{i_{G}(j)+1} a_{+h}(x-h) v(x)-\sum_{i_{L}(j)-1} a_{+h}(x) v(x)-\sum_{\Omega_{h}}\left(a_{+h}(x)-a_{+h}(x-h)\right) v(x) .
\end{aligned}
$$

Hence we have the formula (2.6). Similarly we have

$$
\begin{aligned}
& \sum_{\substack{j_{G}(i) 1+\\
i}} a_{+k}(y-k) v(y)-\sum_{\substack{j_{L}(i)-1 \\
i}} a_{+k}(y) v(y) \\
= & \sum_{\Omega_{h}+\left\{j_{L}(i)-1\right\}} k a_{+k} v_{y}+\sum_{\Omega_{h}}\left(a_{+k}(y)-a_{+k}(y-k)\right) v .
\end{aligned}
$$

Now we define two functions $\psi_{1}$ and $\psi_{2}$ over a region $\Omega^{\prime} \supset \Omega$ which are equal to $\cos (n, x)^{4)}$ and $\cos (n, y)$ respectively at a point on each side of $\Gamma_{h}+\Gamma_{h}^{\prime}$, and which have bounded derivatives of the first order in $\Omega^{\prime}$. It is possible if the boundary $\Gamma$ of $\Omega$ is smooth. The expression to be estimated can be written as follows:

$$
\begin{aligned}
& \sum_{\Gamma_{h}} u^{2} \triangle \Gamma+\sum_{\Gamma_{h}^{\prime}} u^{2} \triangle \Gamma \\
& =\sum_{\substack{i G^{+1} \\
j G+1}} u^{2}\left[\left(\frac{a_{-h}}{\triangle \Gamma}\right)^{2}+\left(\frac{a_{-k}}{\triangle \Gamma}\right)^{2}\right] \triangle \Gamma+\sum_{\substack{i G^{+1} \\
j_{L}-1}} u^{2}\left[\left(\frac{a_{-h}}{\triangle \Gamma}\right)^{2}+\left(\frac{a_{+k}}{\triangle \Gamma}\right)^{2}\right] \triangle \Gamma \\
& +\sum_{\substack{i \\
j_{G}-1}} u^{2}\left[\left(\frac{a_{+h}}{\triangle \Gamma}\right)^{2}+\left(\frac{a_{-k}}{\triangle \Gamma}\right)^{2}\right] \triangle \Gamma+\sum_{\substack{i_{L-1}-1 \\
j_{L}-1}} u^{2}\left[\left(\frac{a_{+h}}{\triangle \Gamma}\right)^{2}+\left(\frac{a_{+k}}{\triangle \Gamma}\right)^{2}\right] \Delta \Gamma \\
& +\sum_{\substack{i_{L^{-1}} \\
j_{L}-1}} u^{2}\left[\left(\frac{a_{+h}-a_{-h}}{\Delta \Gamma}\right)^{2}+\left(\frac{a_{+k}}{\triangle \Gamma}\right)^{2}\right] \triangle \Gamma \\
& +\sum_{\substack{i_{\Delta}+1 \\
\Delta}} u^{2}\left[\left(\frac{a_{-h}}{\triangle \Gamma}\right)^{2}+\left\{\frac{a_{+k}-a_{-k}}{\triangle \Gamma}\right\}_{x-h}^{2}\right] \Delta \Gamma+\sum_{i_{-}-1} u^{2}\left[\left(\frac{a_{+h}}{\triangle \Gamma}\right)^{2}\right. \\
& \left.+\left\{\frac{a_{+k}-a_{-k}}{\triangle \Gamma}\right\}_{x+h}^{2}\right] \triangle \Gamma+
\end{aligned}
$$

4) $\cos (n, x)$ means the direction cosine between the outer normal and the $x$-axis. 


$$
\begin{gathered}
+\sum_{\substack{j_{G}+1 \\
u^{\prime}}} u^{2}\left[\left(\frac{a_{-k}}{\Delta \Gamma}\right)^{2}+\left\{\frac{a_{+h}-a_{-h}}{\triangle \Gamma}\right\}_{y-k}^{2}\right] \triangle \Gamma+\sum_{\substack{j_{L}-1 \\
\triangle}} u^{2}\left[\left(\frac{a_{+k}}{\Delta \Gamma}\right)^{2}\right. \\
\left.+\left\{\frac{a_{+h}-a_{-h}}{\triangle \Gamma}\right\}_{y+k}^{2}\right] \triangle \Gamma \\
+\sum_{\Gamma_{h}^{\prime}} u^{2}\left[\left(\frac{a_{+h}-a_{-h}}{\triangle \Gamma}\right)+\left(\frac{a_{+k}-a_{-k}}{\triangle \Gamma}\right)^{2}\right] \triangle \Gamma
\end{gathered}
$$

where the right side is what was expanded in the case of Fig. 1. The symbols $\sum_{\substack{i_{G+1} \\ j_{G}+1}}$ and $\sum_{\substack{i_{L}-1 \\ j_{L}-1}}^{\prime}$, etc. mean the summation over the boundary mesh points of the first kind facing triangular meshes, and the symbol $\sum_{\substack{i_{L}-1 \\ j_{L}-1}}^{\prime \prime}$ denotes the quantity at the boundary mesh points of the first kind facing a quadrate mesh. Further $\sum_{i_{\Delta}+1}$, etc. denote the summation over the boundary mesh points of the second kind. In other cases we have only trivial change.

Now the last equation becomes

$$
\begin{aligned}
& \sum_{\Gamma_{h}} u^{2} \triangle \Gamma+\sum_{\Gamma_{h}^{\prime}} u^{2} \triangle \Gamma \\
& =\sum_{\substack{\left.i_{G}(j)\right)+1 \\
j}} a_{-h} u^{2}\left(\frac{a_{-h}}{\triangle \Gamma}\right)-\sum_{\substack{i_{L}(j)-1 \\
j}}^{\prime} a_{+h} u^{2}\left(\frac{-a_{+h}}{\triangle \Gamma}\right)-\sum_{\substack{i_{L}-1 \\
j_{L}-1}}^{\prime \prime} a_{+h} u^{2}\left(-\frac{a_{+h}-a_{-h}}{\triangle \Gamma}\right)
\end{aligned}
$$

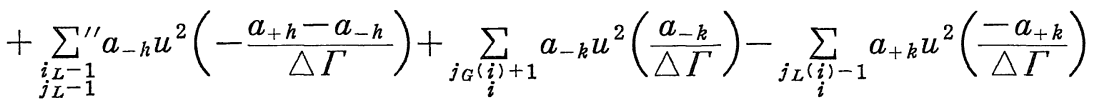

$$
\begin{aligned}
& +\sum_{\substack{i_{\Delta}+1 \\
\Delta}} u^{2}\left\{\frac{a_{+k}-a_{-k}}{\triangle \Gamma}\right\}_{x-h}^{2} \Delta \Gamma+\sum_{\substack{i_{L}-1 \\
\Delta}} u^{2}\left\{\frac{a_{+k}-a_{-k}}{\Delta \Gamma}\right\}_{x+h}^{2} \Delta \Gamma+ \\
& +\sum_{\substack{j_{G}+1 \\
\Delta}} u^{2}\left\{\frac{a_{+h}-a_{-h}}{\triangle \Gamma}\right\}_{y-k}^{2} \Delta \Gamma+\sum_{\substack{j_{L}-1 \\
\Delta}} u^{2}\left\{\frac{a_{+h}-a_{-h}}{\triangle \Gamma}\right\}_{y+k}^{2} \triangle \Gamma \\
& +\sum_{\Gamma_{h}^{\prime}} u^{2}\left[\left(\frac{a_{+h}-a_{-h}}{\Gamma \triangle}\right)^{2}+\left(\frac{a_{+k}-a_{-k}}{\triangle \Gamma}\right)^{2}\right] \triangle \Gamma
\end{aligned}
$$

where $\sum_{i_{L}(j)-1}^{\prime}=\sum_{\substack{i_{L}-1 \\ j_{G}+1}}+\sum_{\substack{i_{L}(j)-1 \\ \Delta}}+\sum_{\substack{i_{L}-1 \\ j_{L}-1}}^{\prime}$ By using the functions $\phi_{1}$ and $\phi_{2}$, we have 


$$
\begin{aligned}
& \sum_{\Gamma_{h}} u^{2} \triangle \Gamma+\sum_{\Gamma_{h}^{\prime}} u^{2} \Delta \Gamma= \\
& =\sum_{i_{G}(j)+1} a_{-h} u^{2} \psi_{1}-\sum_{i_{L}(j)-1} a_{+h} u^{2} \psi_{1}+\sum_{i_{L}-1}^{\prime \prime} a_{-h} u^{2} \psi_{1} \\
& +\sum_{j_{G}(i)+1} a_{-k} u^{2} \psi_{2}-\sum_{j_{L}(i, i)-1} a_{+k} u^{2} \psi_{2} \\
& +\sum_{\substack{i G^{+} \\
\Delta}} u^{2} \phi_{2}^{2} \triangle \Gamma+\sum_{\substack{i_{L}-1 \\
\Delta}} u^{2} \psi_{2}^{2} \triangle \Gamma \\
& +\sum_{\substack{j G^{+} 1 \\
\Delta}} u^{2} \psi_{1}^{2} \triangle \Gamma+\sum_{\substack{j_{L}-1 \\
\Delta}} u^{2} \psi_{1}^{2} \triangle \Gamma+\sum_{\Gamma_{h}^{\prime}} u^{2}\left[\psi_{1}^{2}+\psi_{2}^{2}\right] \triangle \Gamma .
\end{aligned}
$$

By applying (2.6) and (2.7), the last equation becomes

$$
\begin{aligned}
& \text { (2.8) } \sum_{\Gamma_{h}} u^{2} \triangle \Gamma+\sum_{\Gamma_{h}^{\prime}} u^{2} \triangle \Gamma= \\
& =\sum_{\Omega_{h}+\left\{i_{L}(j)-1\right\}} h a_{+h}\left(\psi_{1} u^{2}\right)_{x}+\sum_{\Omega_{h}}\left(a_{+h}(x)-a_{+h}(x-h)\right) \psi_{1} u^{2} \\
& +\sum_{\Omega_{h}+\left\{j_{L}(i)-1\right\}} k a_{+k}\left(\psi_{2} u^{2}\right)_{y}+\sum_{\Omega_{h}}\left(a_{+k}(y)-a_{+k}(y-k)\right) \psi_{2} u^{2} \\
& +\sum_{\substack{i_{L}-1 \\
j_{L}-1}}^{\prime \prime} a_{-h} \psi_{1}\left\{u^{2}-2 k u u_{\bar{y}}+k^{2} u^{2}\right\}_{y+k} \\
& +\sum_{\substack{i_{G}+1 \\
\Delta}} \psi_{2}^{2} u^{2} \triangle \Gamma+\sum_{\substack{i_{L}-1 \\
\triangle}} \psi_{2}^{2} u^{2} \triangle \Gamma \\
& +\sum_{\substack{j G^{+}+1 \\
\Delta}} \psi_{1}^{2} u^{2} \triangle \Gamma+\sum_{\substack{j_{L}-1 \\
\triangle}} \psi_{1}^{2} u^{2} \triangle \Gamma+\sum_{\Gamma_{h}^{\prime}}\left[\psi_{1}^{2}+\psi_{2}^{2}\right] u^{2} \triangle \Gamma \text {. }
\end{aligned}
$$

Here we note that because the equalities $a_{-h}(x)=a_{+h}(x-h)$ and $a_{-k}(y)$ $=a_{+k}(y-k)$ hold at some interior mesh points of $\Omega_{h}$, we have

$$
\begin{gathered}
\sum_{\Omega_{h}}\left(a_{+h}(x)-a_{+h}(x-h)\right) \psi_{1} u^{2} \\
=\left(\sum_{\substack{j G \\
(\Delta)}}+\sum_{\substack{j=\\
(\Delta)}}+\sum_{\Gamma_{h}^{\prime}}+\sum_{\Gamma_{h}^{\prime \prime}}\right)\left(a_{+h}(x)-a_{+h}(x-h)\right) \psi_{1} u^{2}
\end{gathered}
$$

and

$$
\begin{gathered}
\sum_{\Omega_{h}}\left(a_{+k}(y)-a_{+k}(y-k)\right) \psi_{2} u^{2} \\
=\left(\sum_{\substack{i g \\
(\Delta)}}+\sum_{\substack{i_{L} \\
(\Delta)}}+\sum_{\Gamma_{h}^{\prime}}\right)\left(a_{+k}(y)-a_{+k}(y-k)\right) \psi_{2} u^{2},
\end{gathered}
$$

where $\sum_{\substack{i_{G} \\(\Delta)}}\left(\sum_{\substack{i_{L} \\(\Delta)}} \sum_{\substack{j_{G} \\(\Delta)}}\right.$ and $\left.\sum_{\substack{j_{L} \\(\Delta)}}\right)$ denotes the summation over the mesh points $i_{G}\left(i_{L}, j_{G}\right.$ and $\left.j_{L}\right)$ adjoining the boundary mesh point of the second kind. 
$\sum_{\Gamma_{h}^{\prime \prime}}$ denotes the summation over the mesh point adjoining two boundary mesh points of the first kind, one of which faces to a quadrate mesh and another to a triangular mesh. (See the point $\beta$ in Fig. 1). Now

$$
\begin{aligned}
& \sum_{\substack{j_{G} \\
(\Delta)}}\left(a_{+h}(x)-a_{+h}(x-h)\right) \psi_{1} u^{2}=-\sum_{\substack{j_{G} \\
(\Delta)}} \phi_{1}(y+k) \phi_{1}(y) u^{2} \Delta \Gamma \\
=- & \sum_{j_{G}+1} \psi_{1}^{2}\left[u^{2}-2 k u u_{\bar{y}}+k^{2} u_{\bar{y}}^{2}\right] \Delta \Gamma+\sum_{\substack{j_{G} \\
(\Delta)}} k \psi_{1}(y+k) \psi_{1 y}(y) u^{2} \Delta \Gamma, \\
& \sum_{\substack{j_{L} \\
(\Delta)}}\left(a_{+h}(x)-a_{+h}(x-h)\right) \psi_{1} u^{2} \\
=- & \sum_{j_{L}-1} \psi_{1}^{2}\left[u^{2}+2 k u u_{y}+k^{2} u_{y}^{2}\right] \Delta \Gamma-\sum_{\substack{j_{L} \\
(\Delta)}} k \psi_{1}(y-k) \psi_{1 \bar{y}}(y) u^{2} \Delta \Gamma, \\
& \sum_{\substack{i_{G} \\
(\Delta)}}\left(a_{+k}(y)-a_{+k}(y-k)\right) \psi_{2} u^{2} \\
=- & \sum_{\substack{i_{G}+1 \\
\Delta}} \phi_{2}^{2}\left[u^{2}-2 h u u_{\bar{x}}+h^{2} u_{\bar{x}}^{2}\right] \Delta \Gamma+\sum_{\substack{i_{G} \\
(\Delta)}} h \psi_{2}(x+h) \psi_{2 x}(x) u^{2} \Delta \Gamma
\end{aligned}
$$

and

$$
\begin{aligned}
& \sum_{\substack{i_{L} \\
(\Delta)}}\left(a_{+k}(y)-a_{+k}(y-k)\right) \psi_{2} u^{2} \\
=- & \sum_{i_{L}-1} \psi_{2}^{2}\left[u^{2}+2 h u u_{x}+h^{2} u_{x}^{2}\right] \Delta \Gamma-\sum_{\substack{i_{L} \\
(\Delta)}} h \psi_{2}(x-h) \psi_{2 \bar{x}} u^{2} \Delta \Gamma .
\end{aligned}
$$

We note also that

$$
\begin{aligned}
& \sum_{\Gamma_{h}^{\prime}}\left(a_{+h}(x)-a_{+h}(x-h)\right) \psi_{1} u^{2}+\sum_{\Gamma^{h}}\left(a_{+k}(y)-a_{+k}(y-k)\right) \psi_{2} u^{2} \\
= & -\sum_{\Gamma_{h}^{\prime}}\left(\phi_{1}^{2}+\psi_{2}^{2}\right) u^{2} \triangle \Gamma
\end{aligned}
$$

and

$$
\begin{aligned}
& \sum_{\Gamma_{h}^{\prime \prime}}\left(a_{+h}(x)-a_{+h}(x-h)\right) \psi_{1} u^{2}=\sum_{\beta}\left(a_{+h}(x)-a_{+h}(x-h)\right) \psi_{1} u^{2} \\
=- & \sum_{\substack{i_{L}-1 \\
j_{L}-1}}^{\prime \prime} a_{-h} \psi_{1} u(y+k)^{2}-\sum_{\substack{i_{L}-1 \\
j_{L}-1}}^{\prime \prime} a_{-h} k \psi_{1 y} u(y+k)^{2} .
\end{aligned}
$$

Therefore from (2.8), we have

$$
\begin{aligned}
& \sum_{I^{\prime} h} u^{2} \Delta \Gamma+\sum_{T_{h}^{\prime}} u^{2} \Delta \Gamma \\
= & \sum_{\Omega_{l}+\left\{i_{L}(j)-1\right\}} h a_{+h}\left(\phi_{1} u^{2}\right)_{x}+\sum_{\Omega_{h}+\left\{j_{L}(i)-1\right\}} k a_{+k}\left(\phi_{2} u^{2}\right)_{y}+
\end{aligned}
$$




$$
\begin{aligned}
& -\sum_{\substack{i G+1 \\
\triangle}} \psi_{2}^{2}\left[-2 h u u_{\bar{x}}+h^{2} u_{\bar{x}}^{2}\right] \triangle \Gamma+\sum_{\substack{i_{G} \\
(\Delta)}} h \psi_{2}(x+h) \psi_{2 x}(x) u^{2} \triangle \Gamma \\
& -\sum_{\substack{i_{L}-1 \\
\Delta}} \psi_{2}^{2}\left[2 h u u_{x}+h^{2} u_{x}^{2}\right] \triangle \Gamma-\sum_{\substack{i_{L} \\
(\triangle)}} h \psi_{2}(x-h) \psi_{2} \bar{x} u^{2} \triangle \Gamma \\
& -\sum_{\substack{j_{G}+1 \\
\Delta}} \psi_{1}^{2}\left[-2 k u u_{\bar{y}}+k^{2} u_{\bar{y}}^{2}\right] \triangle \Gamma+\sum_{\substack{j_{G} \\
(\triangle)}} k \psi_{1}(y+k) \psi_{1 y}(y) u^{2} \triangle \Gamma \\
& -\sum_{\substack{j_{L}-1 \\
\Delta}} \psi_{1}^{2}\left[2 k u u_{y}+k^{2} u_{y}^{2}\right] \triangle \Gamma-\sum_{\substack{j_{L} \\
(\Delta)}} k \psi_{1}(y-k) \phi_{1 \bar{y}}(y) u^{2} \triangle \Gamma \\
& +\sum_{\substack{i_{L}-1 \\
j_{L}-1}}^{\prime \prime} a_{-h} \psi_{1}\left\{-2 k u u_{\bar{y}}+k^{2} u_{\bar{y}}^{2}\right\}_{y+k}-a_{-h} k \psi_{1 y} u(y+k)^{2} .
\end{aligned}
$$

Applying the formulae

$$
\begin{aligned}
& \left(\phi_{1} u^{2}\right)_{x}=\phi_{1 x} u^{2}+\phi_{1}(x+h) u_{x} u+\left\{\phi_{1} u u_{\bar{x}}\right\}_{x+h}, \\
& \left(\phi_{2} u^{2}\right)_{y}=\phi_{2 y} u^{2}+\phi_{2}(y+k) u_{y} u+\left\{\phi_{2} u u_{\bar{y}}\right\}_{y+k}
\end{aligned}
$$

and using the Schwarz's inequality in the right side of (2.9), we have the estimate

$$
\begin{aligned}
& \sum_{\Gamma_{h}} u^{2} \triangle \Gamma+\sum_{\Gamma_{h}^{\prime}} u^{2} \Delta \Gamma \\
\leqq & \varepsilon \sum_{\Omega_{h}}\left[h\left(a_{+h} u_{x}^{2}+a_{-h} u_{\frac{2}{x}}^{2}\right)+k\left(a_{+k} u_{y}^{2}+a_{-k} u \frac{2}{y}\right)\right]+C(\varepsilon) \sum_{\Omega_{h}} S_{h} u^{2} \\
& +O(h) \sum_{\Gamma_{h}^{\prime}} u^{2} \triangle \Gamma
\end{aligned}
$$

where $C(\varepsilon)$ depends on $\varepsilon$ and the bound of $\psi_{1}, \psi_{2}, \frac{\partial \psi_{1}}{\partial x}, \cdots, \frac{\partial \psi_{2}}{\partial y}$, etc.

From the last equation we get Lemma 1 for small $h$.

Lemma 2. For any $u$ defined on $\Omega_{h} \times[s \triangle t ; s=0,1, \ldots, p, p \triangle t \leqslant T]$, we have the inequalities

$$
\sum_{\Omega_{h}} S_{h} u(p \triangle t)^{2} \leqq 2 T \sum_{s=1}^{p} \Delta t \sum_{\Omega_{h}} S_{h} u \bar{t}(s \triangle t)^{2}+2 \sum_{\Omega_{h}} S_{h} u(0)^{2}
$$

and

(2.11) $\sum_{s=0}^{p} \triangle t \sum_{\Omega_{h}} S_{h} u(s \triangle t)^{2} \leqq 2 T^{2} \sum_{s=1}^{p} \Delta t \sum_{\Omega_{h}} S_{h} u \bar{t}(s \triangle t)^{2}+2 T \sum_{\Omega_{h}} S_{h} u(0)$.

The proof of Lemma 2 is easy. In fact 


$$
\begin{aligned}
& u(p \triangle t)=\sum_{s=1}^{p} \triangle t u_{\bar{t}}(s \triangle t)+u(0), \\
& u(p \triangle t)^{2} \leqslant 2\left(p \triangle t \sum_{s=1}^{p} \triangle t u_{\bar{t}}(s \triangle t)^{2}+u(0)^{2}\right) .
\end{aligned}
$$

Hence we have the desired inequalities (2.10) and (2.11).

Now we return to the inequality (2.5). At first we note that by the boundary conditions

$$
\sum_{\Gamma_{h}} u_{\bar{t}}^{2} \triangle \Gamma \leqq \frac{C_{2}}{\sqrt{h k}} \sum_{\Omega_{h}} S_{h} u_{\bar{t}}^{2}+C_{3} h^{2} \sum_{\Gamma_{h}} g_{\bar{t}}^{2} \triangle \Gamma
$$

Using Lemma 1, Lemma 2 and the last equation, we have the following estimtes about the term of the summation $\sum_{\Gamma_{h}}+\sum_{\Gamma_{h}^{\prime}}$, on the right side of (2.5),

$$
\begin{aligned}
& \left(\sum_{\Gamma_{h}}+\sum_{\Gamma_{h}^{\prime}}\right) \Delta \Gamma\left[\left(1+\frac{3}{2}|\delta|\right) u^{2}+\frac{\Delta t^{2}}{2}(1+|\delta|) u\right] \frac{2}{t} t \Delta t \\
& \leqq\left(1+\frac{3}{2}|\delta|\right) \varepsilon \sum_{\Omega_{h}}\left[h\left(a_{+h} u_{x}^{2}+a_{-h} u_{\bar{x}}^{2}\right)+k\left(a_{+k} u_{y}^{2}+a_{-k} u_{\bar{y}}^{2}\right)\right]_{p \Delta t} \\
& +\left(1+\frac{3}{2}|\delta|\right) C(\varepsilon) \sum_{\Omega_{h}} S_{h} u(p \triangle t)^{2} \\
& +\frac{C_{2} \Delta t^{2}}{2 \sqrt{h k}}(1+|\delta|) \sum_{\Omega_{h}} S_{h} u_{\bar{t}}(p \Delta t)^{2}+\frac{C_{3} \Delta t^{2} h^{2}}{2}(1+|\delta|) \sum_{\Gamma_{h}} g_{\bar{t}}^{2}(p \triangle t) \Delta \Gamma \\
& \leqq \varepsilon^{\prime} \sum_{\Omega_{h}}\left[h\left(a_{+h} u_{x}^{2}+a_{-h} u_{x}^{2}\right)+k\left(a_{+k} u_{y}^{2}+a_{-k} u_{y}^{2}\right)\right]_{p \Delta t} \\
& +C_{4}\left(\varepsilon^{\prime}\right) \sum_{s=1}^{p} \Delta t \sum_{\Omega_{h}} S_{h} u_{\bar{t}}(s \Delta t)^{2} \\
& +C_{5} \frac{\Delta t^{2}}{\sqrt{h k}} \sum_{\Omega_{h}} S_{h} u_{\bar{t}}(p \triangle t)^{2}+C_{4}^{\prime}\left(\varepsilon^{\prime}\right) \sum_{\Omega_{h}} S_{h} u(0)^{2} \\
& +C_{3}^{\prime} \triangle t^{2} h^{2} \sum_{\Gamma_{h}} g \bar{t}(p \triangle t)^{2} \triangle \Gamma
\end{aligned}
$$

where $\quad \varepsilon^{\prime}=\left(1+\frac{3}{2}|\delta|\right) \varepsilon, \quad C_{4}\left(\varepsilon^{\prime}\right)=2 T\left(1+\frac{3}{2}|\delta|\right) C(\varepsilon), C_{5}=\frac{C}{2}(1+|\delta|)$, $C_{4}^{\prime}\left(\varepsilon^{\prime}\right)=2\left(1+\frac{3}{2}|\delta|\right) C(\varepsilon)$ and $C_{3}^{\prime}=\frac{3}{2}(1+|\delta|)$. And 


$$
\begin{aligned}
& \sum_{s=0}^{p-1} \Delta t\left(\sum_{\Gamma_{h}}+\sum_{\Gamma_{h}^{\prime}}\right) u(s \Delta t)^{2} \Delta \Gamma \\
& \leqq \varepsilon \sum_{s=0}^{p-1} \Delta t \sum_{\Omega_{h}}\left[h\left(a_{+h} u_{x}^{2}+a_{-h} u_{\bar{x}}^{2}\right)+k\left(a_{+k} u_{y}^{2}+a_{-k} u z_{\bar{y}}^{2}\right)\right]_{s \Delta t} \\
& \quad+C(\varepsilon) \sum_{s=0}^{p-1} \Delta t \sum_{\Omega_{h}} S_{h} u_{\bar{t}}(s \Delta t)^{2} \\
& \leqq \varepsilon \sum_{s=0}^{p-1} \Delta t \sum_{\Omega_{h}}\left[h\left(a_{+h} u_{x}^{2}+a_{-h} u_{\bar{x}}^{2}\right)+k\left(a_{+k} u_{y}^{2}+a_{-k} u \frac{2}{\bar{y}}\right)\right]_{s \Delta t} \\
& \quad+C_{6}(\varepsilon) \sum_{s=0}^{p-1} \Delta t \sum_{\Omega_{h}} S_{h} u_{\bar{t}}(s \Delta t)^{2}+C_{6}(\varepsilon)^{\prime} \sum_{\Omega_{h}} S_{h} u(0)^{2}
\end{aligned}
$$

where $C_{6}(\varepsilon)=2 T^{2} C(\varepsilon)$ and $C_{6}(\varepsilon)^{\prime}=2 T C(\varepsilon)$. By applying these results in (2.5), the following inequality holds:

$$
\begin{aligned}
& \text { (2.12) }\left\{\sum_{\substack{i_{j}(j) \\
j}}\left[\frac{S_{h}^{\prime}}{2}-\Delta t\left(\frac{a_{+h}}{4}+\frac{a_{-h}}{8}\right)\right] u_{\bar{t}}^{2}+\sum_{\substack{i_{L_{j}^{\prime}(j)} \\
j}}\left[\frac{S_{h}^{\prime}}{2}-\Delta t\left(\frac{a_{-h}}{4}+\frac{a_{+h}}{8}\right)\right] u \frac{2}{t}\right. \\
& +\sum_{j G_{i}^{(i)}}\left[\frac{S_{h}^{\prime}}{2}-\triangle t\left(\frac{a_{+k}}{4}+\frac{a_{-k}}{8}\right)\right] u_{\bar{t}}^{2}+\sum_{j_{L_{i}}(i)}\left[\frac{S_{h}^{\prime}}{2}-\Delta t\left(\frac{a_{-k}}{4}+\frac{a_{+k}}{8}\right)\right] u_{\bar{t}}^{2}
\end{aligned}
$$

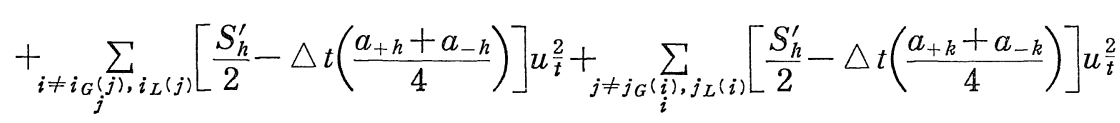

$$
\begin{aligned}
& +\sum_{\substack{i G_{j}^{(j)} \\
j}} h a_{+h}\left(1-\frac{2 \triangle t}{h}-\varepsilon^{\prime}\right) u_{x}^{2}+\frac{1}{2} \sum_{\substack{i \neq i_{G}(j) \\
j}} h a_{+h}\left(1-\frac{2 \triangle t}{h}-2 \varepsilon^{\prime}\right) u_{x}^{2} \\
& +\sum_{\left.i_{j}^{\prime \prime} j\right)} h a_{-h}\left(1-\frac{2 \triangle t}{h}-\varepsilon^{\prime}\right) u_{\bar{x}}^{2}+\frac{1}{2} \sum_{\substack{i \neq i_{j}(j) \\
j}} h a_{-h}\left(1-\frac{2 \triangle t}{h}-2 \varepsilon^{\prime}\right) u_{x}^{\frac{2}{x}} \\
& +\sum_{\substack{G_{i}(i) \\
i}} k a_{+k}\left(1-\frac{2 \triangle t}{k}-\varepsilon^{\prime}\right) u_{y}^{2}+\frac{1}{2} \sum_{j \neq j_{i}(i)} k a_{+k}\left(1-\frac{2 \Delta t}{k}-2 \varepsilon^{\prime}\right) u_{y}^{2} \\
& \left.+\sum_{j_{i}^{\prime}(i)} k a_{-k}\left(1-\frac{2 \triangle t}{k}-\varepsilon^{\prime}\right) u \frac{2}{y}+\frac{1}{2} \sum_{j \neq j_{i}(i)} k a_{-k}\left(1-\frac{2 \Delta t}{k}-2 \varepsilon^{\prime}\right) u \frac{2}{\bar{y}}\right\}_{p \triangle t} \\
& \leqq C_{7}(\varepsilon) \sum_{s=1}^{p} \Delta t \sum_{\Omega_{h}} S_{h} u_{\bar{t}}(s \Delta t)^{2} \\
& +\varepsilon \sum_{s=1}^{p} \Delta t \sum_{\Omega_{h}}\left[h\left(a_{+h} u_{x}^{2}+a_{-h} u_{\bar{x}}^{2}\right)+k\left(a_{+k} u_{y}^{2}+a_{-k} u_{\frac{y}{y}}^{2}\right)\right]_{s \triangle t} \\
& +F(p)+G(p)+\Phi
\end{aligned}
$$


where

$$
\begin{aligned}
S_{h}^{\prime}=S_{h} & \left(1-C_{1} \triangle t-C_{5} \frac{\Delta t^{2}}{\sqrt{h k}}\right), C_{7}(\varepsilon)=1+C_{4}\left(\varepsilon^{\prime}\right)+C_{6}(\varepsilon), \\
F(p)= & \sum_{s=1}^{p-1} \Delta t \sum_{\Omega_{h}} S_{h} \bar{f}^{2}, \\
G(p)= & \left(\sum_{\Gamma_{h}}+\sum_{\Gamma_{h}^{\prime}}\right) \triangle \Gamma\left[2 \bar{g}((p-1) \triangle t)^{2}+\Delta t^{2}\left(1+\frac{C_{2} \sqrt{h k}}{2}\right) g_{\bar{t}}(p \Delta t)^{2}\right] \\
& +\sum_{s=0}^{p-1} \Delta t\left(\sum_{\Gamma_{h}}+\sum_{\Gamma_{h}^{\prime}}\right) \triangle \Gamma \bar{g}_{t}^{2}+\frac{1}{2}\left(\sum_{\Gamma_{h}}+\sum_{\Gamma_{h}^{\prime}}\right) \triangle \Gamma\left[\bar{g}(0)^{2}+\right. \\
& \left.+(1+\triangle t) \bar{g}(\triangle t)^{2}\right]+C_{3}^{\prime} \triangle t^{2} h^{2} \sum_{\Gamma_{h}} g_{\bar{t}}(p \Delta t)^{2} \triangle \Gamma
\end{aligned}
$$

and

$$
\begin{aligned}
& \Phi=\frac{1}{2}\left\{\left(\sum_{\substack{i_{G}(j) \\
j}}+\sum_{\Omega_{h}}\right) h a_{+h}\left[\left(1+\frac{\Delta t}{2}\right) \varphi_{x}^{2}+\frac{\Delta t}{2} \psi_{x}^{2}\right]\right. \\
& +\left(\sum_{\substack{i_{L}(j) \\
j}}+\sum_{\Omega_{h}}\right) h a_{-h}\left[\left(1+\frac{\Delta t}{2}\right) \varphi_{\frac{2}{x}}+\frac{\Delta t}{2} \psi_{x}^{2}\right] \\
& +\left(\sum_{\substack{j G_{i}(i) \\
\Omega^{\prime}}}+\sum_{\Omega_{h}}\right) k a_{+k}\left[\left(1+\frac{\Delta t}{2}\right) \varphi_{y}^{2}+\frac{\Delta t}{2} \psi_{y}^{2}\right] \\
& \left.+\left(\sum_{j_{L_{i}}(i)}+\sum_{\Omega_{h}}\right) k a_{-k}\left[\left(1+\frac{\Delta t}{2}\right) \varphi_{y}^{\frac{2}{y}}+\frac{\Delta t}{2} \psi_{y}^{\frac{2}{y}}\right]\right\} \\
& +\sum_{\Omega_{h}} S_{h} \psi^{2}+\left(C_{4}\left(\varepsilon^{\prime}\right)+C_{6}(\varepsilon)^{\prime}\right) \sum_{\Omega_{h}} S_{h} \varphi^{2} \\
& +\frac{1}{2}\left(\sum_{\Gamma_{h}}+\sum_{\Gamma_{h}^{\prime}}\right) \Delta \Gamma\left[\varphi^{2}+\Delta t \psi^{2}\right] \text {. }
\end{aligned}
$$

Now we choose $\triangle t, h$ and $k$ as follows:

$$
1-\frac{2 \triangle t}{h} \geqq 2 \alpha>0,1-\frac{2 \triangle t}{k} \geqq 2 \alpha>0
$$

where $\alpha$ is a small constant. Then for sufficiently small $\Delta t$

$$
\frac{S_{h}^{\prime}}{2}-\Delta t\left(\begin{array}{c}
a_{\lrcorner} h \\
4
\end{array}+\frac{a_{-h}}{8}\right)=\frac{S_{h}}{2}-\frac{\Delta t\left(a_{+h}+a_{-h}\right)}{4}+\Delta t\left(\frac{a_{-h}}{8}-\frac{C_{1} S_{h}}{2}-\frac{C_{5} S_{h} \Delta t}{2 \sqrt{h k}}\right)
$$




$$
\geqq \frac{S_{h}}{2}\left(1-\frac{2 \triangle t}{h} \frac{a_{+k}+a_{-k}}{h}\right) \geqq \alpha S_{h}
$$

etc.,

and

$$
\begin{aligned}
\frac{S_{h}^{\prime}}{2}-\Delta t\left(\frac{a_{+h}+a_{-h}}{4}\right) & \geqq \frac{S_{h}}{2}\left(1-\frac{2 \triangle t}{h} \frac{h}{a_{+k}+a_{-k}}\right)-\frac{S_{h}}{2}\left(C_{1} \Delta t+C_{5} \frac{\Delta t^{2}}{\sqrt{h k}}\right) \\
& \geqq \frac{\alpha}{2} S_{h}
\end{aligned}
$$

etc.

Further we can take $\varepsilon^{\prime}(\varepsilon)$ so small that

$$
h a_{+h}\left(1-\frac{2 \triangle t}{h}-\varepsilon^{\prime}\right) \geqq \alpha h a_{+h}
$$

Substituting these inequalities into (2.12) we have

$$
\begin{aligned}
& \quad \alpha \sum_{\Omega_{h}} S_{h} u_{\bar{t}}(p \Delta t)^{2}+\frac{\alpha}{2} \sum_{\Omega_{h}}\left[h\left(a_{+h} u_{x}^{2}+a_{-h} u_{\bar{x}}^{2}\right)+k\left(a_{+k} u_{y}^{2}+a_{-k} u_{\bar{y}}^{2}\right)\right]_{p \triangle t} \\
& \leqq C_{7}(\varepsilon) \sum_{s=1}^{p} \Delta t \sum_{\Omega_{h}} S_{h} u_{\bar{t}}(s \Delta t)^{2} \\
& \quad+\varepsilon \sum_{s=1}^{p} \Delta t \sum_{\Omega_{h}}\left[h\left(a_{+h} u_{x}^{2}+a_{-h} u_{\bar{x}}^{2}\right)+k\left(a_{+k} u_{y}^{2}+a_{-k} u_{\bar{y}}^{2}\right)\right]_{s \triangle t} \\
& \quad+F(p)+G(p)+\Phi .
\end{aligned}
$$

We set

$$
\begin{aligned}
\sum_{s=1}^{p} & \triangle t \sum_{\Omega_{h}}\left[S_{h} u_{t}^{2}+\frac{h}{2}\left(a_{+h} u_{x}^{2}+a_{-h} u_{x}^{2}\right)+\frac{k}{2}\left(a_{+k} u_{y}^{2}+a_{-k} u_{\bar{y}}^{2}\right)\right]_{s \Delta t} \\
= & S(p) .
\end{aligned}
$$

Then the last inequality can be written in the form

$$
\frac{S(p)-S(p-1)}{\triangle t} \leqq C_{8} S(p)+\Psi(p)
$$

where $\quad C=\max \left(\frac{C_{7}(\varepsilon)}{\alpha}, \frac{2 \varepsilon}{\alpha}\right), \Psi(p)=\frac{1}{\alpha}(F(p)+G(p)+\Phi)$.

Hence we find that

$$
S(p) \leqq \frac{1}{1-C_{8} \Delta t} S(p-1)+\frac{\triangle t}{1-C_{8} \Delta t} \Psi(p) .
$$


We choose $\Delta t$ so small that $1-C_{8} \Delta t \geqq \frac{1}{2}$ and we put $\frac{1}{1-C_{8} \Delta t}=R$.

Then

$$
S(p) \leqq R S(p-1)+R \triangle t \Psi(p)
$$

We apply the last inequality successively, and then

$$
S(p) \leqq R^{p-1} S(1)+R \triangle t \sum_{s=0}^{p} R^{p-1} \Psi(s)
$$

However

$$
R^{p}=\left(1+\frac{C_{8} \Delta t}{1-C_{8} \Delta t}\right)^{p} \leqq e^{\frac{C_{8} p \Delta t}{1-C_{8} \Delta t} \leqq e^{2 C_{8} T}}
$$

since

$$
C_{8} \triangle t \leqq \frac{1}{2}, p \triangle t \leqq T
$$

Therefore we get

$$
S(p) \leqq e^{2 C_{8} T}[S(1)+T \Psi(p)] .
$$

Finally we estimate $S(1)$;

$$
\begin{array}{r}
S(1)=\sum_{\Omega_{h}}\left[S_{h} \psi^{2}+\frac{h}{2} a_{+h}\left(\varphi_{x}+\Delta t \psi_{x}\right)^{2}+\frac{h}{2} a_{-h}\left(\varphi_{\bar{x}}+\Delta t \psi_{\bar{x}}\right)^{2}+\right. \\
\left.+\frac{k}{2} a_{+k}\left(\varphi_{y}+\Delta t \psi_{y}\right)^{2}+\frac{k}{2} a_{-k}\left(\varphi_{\bar{y}}+\Delta t \psi_{\bar{y}}\right)^{2}\right] \\
\leqq \sum_{\Omega_{h}}\left[S_{h} \psi^{2}+\frac{3 h a_{+h}}{2}\left\{\varphi_{x}^{2}+\left(\frac{\triangle t}{h}\right)^{2} \psi(x+h)^{2}+\left(\frac{\triangle t}{h}\right)^{2} \psi(x)^{2}\right\}\right. \\
+\frac{3 h a_{-h}}{2}\left\{\varphi_{x}^{2}+\left(\frac{\triangle t}{h}\right)^{2} \psi(x-h)^{2}+\left(\frac{\triangle t}{h}\right)^{2} \psi(x)^{2}\right\} \\
+\frac{3 k a_{+k}}{2}\left\{\varphi_{y}^{2}+\left(\frac{\triangle t}{k}\right)^{2} \psi(y+k)^{2}+\left(\frac{\triangle t}{k}\right)^{2} \psi(y)^{2}\right\} \\
\left.+\frac{3 k a_{-k}}{2}\left\{\varphi_{\frac{2}{y}}+\left(\frac{\triangle t}{k}\right)^{2} \psi(y-k)^{2}+\left(\frac{\triangle t}{k}\right)^{2} \psi(y)^{2}\right\}\right]
\end{array}
$$




$$
\leqq C_{9} \sum_{\Omega_{h}} S_{h}\left(\psi^{2}+\varphi_{x}^{2}+\varphi_{\frac{2}{x}}^{2}+\varphi_{y}^{2}+\varphi \frac{2}{y}\right)
$$

where we used the fact that the functions $\varphi$ and $\phi$ are smooth in $\Omega^{\prime} \supset \Omega$.

Consequently we have

$$
S(p) \leqq C_{10}(T) \Psi(p)
$$

i. e., $\quad \sum_{s=1}^{p} \Delta t \sum_{\Omega_{h}}\left[S_{h} u \frac{2}{t}+\frac{h}{2}\left(a_{+h} u_{x}^{2}+a_{-h} u \frac{2}{x}\right)+\frac{k}{2}\left(a_{+k} u_{y}^{2}+a_{-k} u \frac{2}{y}\right)\right] s \Delta t$

$$
\leqq C_{10}(T) \Psi(p) \text {. }
$$

From the last inequality and (2.13) we have also a strong inequality

$$
\begin{gathered}
\sum_{\Omega_{h}}\left[S_{h} u \frac{2}{t}+\frac{h}{2}\left(a_{+h} u_{x}^{2}+a_{-h} u \frac{2}{x}\right)+\frac{k}{2}\left(a_{+k} u_{y}^{2}+a_{-k} u \frac{2}{y}\right)\right] p \Delta t \\
\leqq C_{11}(T) \Psi(p) .
\end{gathered}
$$

Thus we have

Theorem 1 (Stability). Suppose that $\Delta t, h$ and $k$ satisfy the condition

$$
1-\frac{2 \Delta t}{h} \geqq \alpha_{1}>0, \quad 1-\frac{2 \Delta t}{k} \geqq \alpha_{1}>0
$$

where $\alpha_{1}$ is an arbitrarily small constant. Then the solution $u$ of the difference problem (1.9), (1.7), (1.10), (1.11) and (1.12) satisfies an energy inequality (2.14) for sufficiently small $\triangle t$ and $p \triangle t \leqq T$.

Immediately from the last theorem we have a convergence theorm. In fact we express the solution of the differential problem (1.1), (1.2) and (1.3) by $v$ and the corresponding solution of the difference problem (1.9), (1.7), (1.10), (1.11), and (1.12) by $u$. And we put $w=v-u$. Let us consider, for example, again the hatched mesh in Fig. 1. Then we have

$$
\begin{aligned}
& S_{h} v_{t \bar{t}}=\iint \frac{\partial^{2} v}{\partial t^{2}} d x d y+M_{1} h S_{h}, \\
& \left(\bar{g}+\delta\{v\}_{x+h}\right)-a_{-h} v_{\bar{x}}+a_{+k} v_{y}-a_{-k} v_{\bar{y}}=\oint \frac{\partial v}{\partial n} d \Gamma+\sum_{i} h M_{2 i} \triangle \Gamma_{i}, \\
& S_{h} \bar{f}=\iint f d x d y,
\end{aligned}
$$


under the condition that $\frac{\partial^{3} v}{\partial t^{3}}, \frac{\partial^{3} v}{\partial x \partial t^{2}}, \frac{\partial^{3} v}{\partial y \partial t^{2}}, \frac{\partial^{2} v}{\partial x^{2}}, \frac{\partial^{2} v}{\partial y^{2}}, \frac{\partial^{2} v}{\partial x \partial y}, \frac{\partial v}{\partial x}$ and $\frac{\partial v}{\partial y}$ are continuous, where $M_{1}$ depends on the magnitude of $\frac{\partial^{3} v}{\partial t^{3}}, \frac{\partial^{3} v}{\partial x \partial t^{2}}$ and $\frac{\partial^{3} v}{\partial y \partial t^{2}}, M_{2 i}$ depend on that of $\frac{\partial^{2} v}{\partial x^{2}}, \frac{\partial^{2} v}{\partial y^{2}}, \frac{\partial^{2} v}{\partial x \partial y}, \frac{\partial v}{\partial x}$ and $\frac{\partial v}{\partial y}$, and $\triangle \Gamma_{i}$ are lengths of sides of the trapezoids. Hence we have

$$
\begin{aligned}
& S_{h} v_{t \bar{t}}-\left[\left(\bar{g}+\delta\{v\}_{x+h}\right)-a_{-h} v_{\bar{x}}+a_{+k} v_{y}-a_{-k} v_{\bar{y}}\right]-S_{h} \bar{f} \\
= & \iint \frac{\partial^{2} v}{\partial t^{2}} d x d y-\oint \frac{\partial v}{\partial n} d \Gamma-\iint f d x d y+M_{1} h S_{h}-\sum_{i} h M_{2 i} \triangle \Gamma_{i} \\
= & \iint\left[\frac{\partial^{2} v}{\partial t^{2}}-\frac{\partial^{2} v}{\partial x^{2}}-\frac{\partial^{2} v}{\partial y^{2}}-f\right] d x d y+M_{1} h S_{h}-\sum_{i} h M_{2 i} \triangle \Gamma_{i} \\
= & M_{1} h S_{h}-\sum_{i} h M_{2 i} \Delta \Gamma_{i},
\end{aligned}
$$

since $v$ is the solution of (1.1). Therefore on the concerned mesh point $w$ satisfies the equation

$$
S_{h} w_{t \bar{t}}=\delta\{w\}_{x+h}-a_{-h} w_{\bar{x}}+a_{+k} w_{y}-a_{-k} w_{\bar{y}}+M_{1} h S_{h}-\sum_{i} h M_{2 i} \triangle \Gamma_{i},
$$

which can be written in the form

$$
S_{h} w_{t \bar{t}}=\left(g_{1}+\delta\{w\}_{x+h}\right)-a_{-h} w_{\bar{x}}+a_{+k} w_{y}-a_{-k} w_{\bar{y}}+S_{h} f_{1}
$$

where $\left|g_{1}\right| \leq$ const. $h$ and $f_{1}=M_{1} h$. Analogous difference equations are satisfied by $w$ on the mesh points adjoining to the boundary mesh point of second kind.

In the same way we have the equation satisfied by $w$ at the interior mesh point;

$$
S_{h} w_{t \bar{t}}=a_{+h} w_{x}-a_{-h} w_{\bar{x}}+a_{+k} w_{y}-a_{-k} w_{\bar{y}}+S_{h} f_{1}, f_{1}=M_{3} h
$$

where $M_{3}$ depends on the magnitude of the third derivatives of $v$ with respect to $t, x$ and $y$.

Therefore we have the difference equations over the region $\Omega_{h}$ in the 
same form as in (1.9);

$$
S_{h} w_{t \bar{t}}=a_{+h} w_{x}-a_{-h} w_{\bar{x}}+a_{+k} w_{y}-a_{-k} w_{\bar{y}}+\delta_{h}\left(g_{1}+\delta w\right) \triangle \Gamma+S_{h} f_{1}
$$

under the boundary conditions in the form as in (1.7);

$$
\begin{array}{ll}
a_{-h} w_{\bar{x}}=\left(g_{1}+\delta w\right) \Delta \Gamma, a_{-h}=k & \text { on a right boundary mesh point, } \\
a_{+h} w_{x}=-\left(g_{1}+\delta w\right) \Delta \Gamma, a_{+h}=k & \text { on a left boundary mesh point, } \\
a_{-k} w_{\bar{y}}=\left(g_{1}+\delta w\right), a_{-k}=h & \text { on an upper boundary mesh point, } \\
a_{+k} w_{y}=-\left(g_{1}+\delta w\right), a_{+k}=h & \text { on a lower boundary mesh point. }
\end{array}
$$

At the boundary point of first kind, the formulae (1.10) and (1.11) approximate formally the original boundary condition $\frac{\partial u}{\partial n}-\delta u=g$ with the error of order $O(h)$. This means

$$
\begin{aligned}
& a_{-h} w_{\bar{x}}+a_{-k} w_{\bar{y}}=\left(g_{1}+\delta w\right) \quad \text { at a right upper mesh point, } \\
& a_{-h} w_{\bar{x}}-a_{+k} w_{y}=\left(g_{1}+\delta w\right) \quad \text { at a right lower mesh point, } \\
& -a_{+h} w_{x}+a_{-k} w_{\bar{y}}=\left(g_{1}+\delta w\right) \quad \text { at a left upper mesh point, } \\
& -a_{+h} w_{x}-a_{+k} w_{y}=\left(g_{1}+\delta w\right) \quad \text { at a left lower mesh point }
\end{aligned}
$$

and (in place of (1.11))

$$
-a_{+h} w_{x}+a_{-h}\left\{w_{\bar{x}}\right\}_{y+k}-a_{+k} w_{y}=\left(g_{1}+\delta w\right) \triangle \Gamma
$$

In all the above formulae about $w$ we can recognize that $g_{1}=O(h), f_{1}=$ $O(h)$. So that we find that $w$ satisfies the difference problem with $\varphi=0$, $\psi=O(\triangle t), g=O(h)$ and $f=O(h)+O\left(\Delta t^{2}\right)$. Therefore we have from (2.14), using Lemma 1 ,

$$
\|w\|_{h}=\|v-u\|_{h}=O(h) \quad \text { when } h \rightarrow 0
$$


uniformly for $p \triangle t \leqslant T$, where

$$
\|w\|_{h}=\sum_{\Omega_{h}}\left[S_{h} w_{t}^{2}+\frac{h}{2}\left(a_{+h} w_{x}^{2}+a_{-h} w_{\bar{x}}^{2}\right)+\frac{k}{2}\left(a_{+k} w_{y}^{2}+a_{-k} w \frac{2}{y}\right)+S_{h} w^{2}\right]
$$

and we supposed that a solution of the differential problem has continuous third-derivatives in $\bar{\Omega} \times[0, T]$. Hence we have

Theorem 2 (Convergence). Suppose that the boundary $\Gamma$ of $\Omega$ is sufficiently smooth and a solution of the differential problem (1.1), (1.2) and (1.3) has the regularity just stated. When $\Delta t, h$ and $k$ tend to zero under the condition (2.15), a solution of our difference problem converges to the corresponding solution of the differential problem in the sense of (2.16).

\section{§3. A Parabolic Problem}

We can construct an analogous difference scheme for a mixed initialboundary problem for an equation of heat conduction

$$
\begin{array}{ll}
\frac{\partial u}{\partial t}-\frac{\partial^{2} u}{\partial x^{2}}-\frac{\partial^{2} u}{\partial y^{2}}=f & \text { in } Q(T), \\
\frac{\partial u}{\partial n}-\delta u=g, & \text { on } \Gamma \times[0, T],^{5)} \\
u(0, x, y)=\varphi(x, y) . &
\end{array}
$$

Here the notations are same as in $\S 1$. As an approximation of the differential equation (3.1) we set an implicit difference equation analogous to (1.9),

$$
S_{h} u_{\bar{t}}=a_{+h} u_{x}-a_{-h} u_{\bar{x}}+a_{+k} u_{y}-a_{-k} u_{\bar{y}}+\delta_{h}(\bar{g}+\delta u) \Delta \Gamma+S_{h} \bar{f} .
$$

As boundary conditions we use the formulae (1.7), (1.10) and (1.11). As initial conditions we use

5) As in $\S 1$, we assume here and in $\S 4$ that at every point $P \in \Gamma$ there is a circle $S$ such that $\bar{S} \cap \bar{\Omega}=P$. 


$$
u(0)=\varphi
$$

In the same way as in $\S 2$ we can prove a stability theorem and a convergence theorem. For the proof we multiply the equation (3.4) by $u \Delta t$ and sum all over $\Omega_{h} \times[t=s \Delta t ; s=1,2, \cdots, p]$, then

$$
\begin{aligned}
& \sum_{s=1}^{p} \Delta t \sum_{\Omega_{h}} S_{h} u \bar{t} u \\
& =\frac{1}{2} \sum_{s=1}^{p} \Delta t \sum_{\Omega_{h}}\left[h\left(a_{+h} u_{x}\right)_{\bar{x}} u+h\left(a_{-h} u_{\bar{x}}\right)_{x} u+k\left(a_{+k} u_{y}\right)_{\bar{y}} u+k\left(a_{-k} u_{\bar{y}}\right)_{y} u\right] \\
& \quad+\sum_{s=1}^{p} \Delta t \sum_{\Omega_{h}}\left[\delta_{h}(\bar{g}+\delta u) u \triangle \Gamma+S_{h} \bar{f} u\right] .
\end{aligned}
$$

However we have

$$
\sum_{s=1}^{p} \Delta t u_{\bar{t}} u=\frac{1}{2} \sum_{s=1}^{p} \Delta t^{2} u_{t}^{2}+\frac{1}{2} u(p \triangle t)^{2}-\frac{1}{2} u(0)^{2}
$$

and

$$
\begin{aligned}
& \sum_{\Omega_{h}} h\left(a_{+h} u_{x}\right)_{\bar{x}} u=\sum_{\Omega_{h}} h\left[\left(a_{+h} u_{x} u\right)_{\bar{x}}-\left\{a_{-h} u_{x}^{2}\right\}_{x-h}\right] \\
& =\sum_{\substack{i_{G}(j) \\
j}} a_{+h} u_{x} u-\sum_{\substack{i_{L}(j)-1 \\
j}} a_{+h} u_{x} u-\sum_{\Omega_{h}} h a_{-h} u_{\bar{x}}^{2} \\
& =\sum_{\substack{i_{G}(j)+1 \\
j}} a_{-h} u_{\bar{x}} u-\sum_{i_{L}(j)-1} a_{j} a_{+h} u_{x} u-\sum_{\substack{i G_{j}(j) \\
j}} h a_{+h} u_{x}^{2}-\sum_{\Omega_{h}} h a_{-h} u_{\bar{x}}^{2}, \\
& \sum_{\Omega_{h}} h\left(a_{-h} u_{x}\right)_{\bar{x}} u \\
& =\sum_{\substack{i_{G}(j)+1 \\
j}} a_{-h} u_{\bar{x}} u-\sum_{i_{L}\langle(j)-1} a_{j} a_{+h} u_{x} u-\sum_{i_{j}(j)} h a_{-h} u_{\bar{x}}^{2}-\sum_{\Omega_{h}} h a_{+h} u_{x}^{2}, \\
& \sum_{\Omega_{h}} k\left(a_{+k} u_{y}\right)_{\bar{y}} u
\end{aligned}
$$

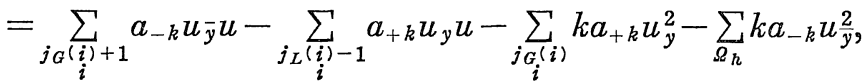

$$
\begin{aligned}
& \sum_{\Omega_{h}} k\left(a_{-k} u_{\bar{y}}\right)_{y} u \\
& =\sum_{j_{G}(i)+1} a_{-k} u \bar{y} u-\sum_{j_{L}(i)-1} a_{+k} u_{y} u-\sum_{j_{L_{i}}(i)} k a_{-k} u \frac{2}{y}-\sum_{\Omega_{k}} k a_{+k} u_{y}^{2} .
\end{aligned}
$$


Therefore the equation (3.6) can be written in the form

$$
\begin{aligned}
& \frac{1}{2} \sum_{\Omega_{h}} S_{h}\left\{u(p \triangle t)^{2}-u(0)^{2}\right\}+\frac{\triangle t}{2} \sum_{s=1}^{p} \triangle t \sum_{\Omega_{h}} S_{h} u_{t}^{2}
\end{aligned}
$$

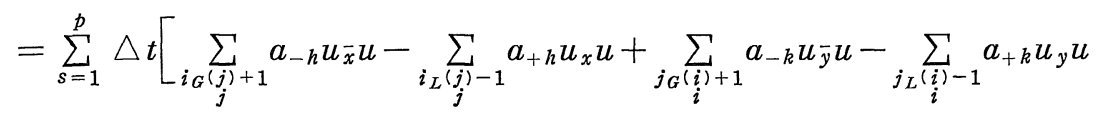

$$
\begin{aligned}
& -\frac{1}{2}\left(\sum_{\Omega_{h}}+\sum_{\substack{i_{G}(j) \\
j}}\right) h a_{+h} u_{x}^{2}-\frac{1}{2}\left(\sum_{\Omega_{h}}+\sum_{i_{L_{j}^{\prime}(j)}}\right) h a_{-h} u_{x}^{2}
\end{aligned}
$$

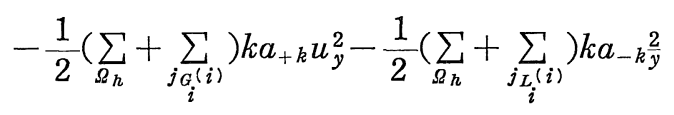

$$
\begin{aligned}
& \left.+\sum_{\Gamma_{h}^{\prime}}(\bar{g}+\delta u) u \Delta \Gamma+\sum_{\Omega_{h}} S_{h} \bar{f} u\right] .
\end{aligned}
$$

Applying the boundary conditions (1.7), (1.10) and (1.11) to the last equation we have

$$
\begin{aligned}
& \frac{1}{2} S_{h} u(p \Delta t)^{2}+\frac{\triangle t}{2} \sum_{s=1}^{p} \Delta t \sum_{\Omega_{h}} S_{h} u_{t}^{\frac{2}{t}} \\
& +\frac{1}{2} \sum_{s=1}^{p} \Delta t\left[\left(\sum_{\Omega_{h}}+\sum_{i_{G_{j}(j)}}\right) h a_{+h} u_{x}^{2}+\left(\sum_{\Omega_{h}}+\sum_{i_{L_{j}(j)}}\right) h a_{-h} u \frac{2}{x}+\right. \\
& +\left(\sum_{\Omega_{h}}+\sum_{j_{i}^{(i)}}\right) k a_{+k} u_{y}^{2}+\left(\sum_{\Omega_{h}}+\sum_{j_{i}(i)} k a_{-k} u \frac{2}{y}\right] \\
& =\frac{1}{2} \sum_{\Omega_{h}} S_{h} u(0)^{2}+\sum_{s=1}^{p} \Delta t\left[\sum_{\Gamma_{h}} \Delta \Gamma(\bar{g}+\delta u) u+\sum_{\Gamma_{h}^{\prime}} \Delta \Gamma(\bar{g}+\delta u) u\right. \\
& \left.+\sum_{\Omega_{h}} S_{h} \bar{f} u\right]
\end{aligned}
$$

Now we estimate the second sum of the right side by the Schwarz's inequality and Lemma 1:

$$
\begin{aligned}
& \left|\sum_{s=1}^{p} \Delta t\left[\sum_{\Gamma_{h}} \Delta \Gamma(\bar{g}+\delta u) u+\sum_{\Gamma_{h}^{\prime}} \Delta \Gamma(\bar{g}+\delta u) u\right]\right| \\
& \leqq \sum_{s=1}^{p} \Delta t\left(\sum_{\Gamma_{h}}+\sum_{\Gamma_{h}^{\prime}}\right) \Delta \Gamma\left[\frac{1}{2} \bar{g}^{2}+\left(\frac{1}{2}+|\delta|\right) u^{2}\right]
\end{aligned}
$$




$$
\begin{aligned}
& \leqq \frac{1}{2} \sum_{s=1}^{p} \triangle t\left(\sum_{\Gamma_{h}}+\sum_{\Gamma_{h}^{\prime}}\right) \bar{g}^{2} \Delta \Gamma \\
& \quad+\left(\frac{1}{2}+|\delta|\right) \varepsilon \sum_{n=1}^{p} \Delta t \sum_{\Omega_{h}}\left[h\left(a_{+h} u_{x}^{2}+a_{-h} u_{\frac{2}{x}}^{2}\right)+k\left(a_{+k} u_{y}^{2}+a_{-k} u \frac{2}{y}\right)\right] \\
& \quad+\left(\frac{1}{2}+|\delta|\right) C(\varepsilon) \sum_{s=1}^{p} \Delta t \sum_{\Omega_{h}} S_{h} u^{2}
\end{aligned}
$$

and

$$
\left|\sum_{s=1}^{p} \triangle t \sum_{\Omega_{h}} S_{h} \bar{f} u\right| \leqq \frac{1}{2} \sum_{s=1}^{p} \triangle t \sum_{\Omega_{h}} S_{h}\left(\bar{f}^{2}+u^{2}\right) .
$$

If we take $\varepsilon$ so small that

$$
\left(\frac{1}{2}+|\delta|\right) \varepsilon \leqq \frac{1}{4}
$$

then we have from (3.7) using the above estimates,

$$
\begin{gathered}
\sum_{\Omega_{h}} S_{h} u(p \Delta t)^{2}+\frac{1}{2} \sum_{s=1}^{p} \Delta t \sum_{\Omega_{h}}\left[h\left(a_{+h} u_{x}^{2}+a_{-h} u \frac{2}{x}\right)+k\left(a_{+k} u_{y}^{2}+a_{-k} u \frac{2}{y}\right)\right] \\
\leqq \sum_{\Omega_{h}} S_{h} u(0)^{2}+C_{1} \sum_{s=1}^{p} \Delta t \sum_{\Omega_{h}} S_{h} u^{2} \\
\quad+\sum_{s=1}^{p} \Delta t\left(\sum_{\Gamma_{h}}+\sum_{\Gamma_{h}^{\prime}}\right) \bar{g}^{2} \Delta \Gamma+\sum_{s=1}^{p} \Delta t \sum_{\Omega_{h}} S_{h} \bar{f}^{2}
\end{gathered}
$$

where $C_{1}=1+(1+2|\delta|) C(\varepsilon)$. Further if we put

$$
\begin{aligned}
S(p) & =\sum_{s=1}^{p} \Delta t \sum_{\Omega_{h}} S_{h} u(s \Delta t)^{2} \\
& +\frac{1}{2} \sum_{q=1}^{p} \Delta t \sum_{s=1}^{q} \Delta t \sum_{\Omega_{h}}\left[h\left(a_{+h} u_{x}^{2}+a_{-h} u \frac{2}{x}\right)+k\left(a_{+k} u_{y}^{2}+a_{-k} u \frac{2}{y}\right)\right] \\
\Psi(p) & =\sum_{\Omega_{h}} S_{h} u(0)^{2}+\sum_{s=1}^{p} \Delta t\left(\sum_{\Gamma_{h}}+\Gamma \Gamma_{h}^{\prime}\right) \bar{g}^{2} \Delta \Gamma+\sum_{s=1}^{p} \Delta t \sum_{\Omega_{h}} S_{h} \bar{f}^{2},
\end{aligned}
$$

we have from (3.8)

$$
\frac{S(p)-S(p-1)}{\triangle t} \leqq C_{1} S(p)+\Psi(p)
$$

By the same way as in $\S 2$ we get

$$
S(p) \leqq C_{2} \Psi(p),
$$


and by (3.9) we have consequently

$$
\begin{gathered}
\sum_{\Omega_{h}} S_{h} u(p \Delta t)^{2}+\frac{1}{2} \sum_{s=1}^{p} \Delta t \sum_{\Omega_{h}}\left[h\left(a_{+h} u_{x}^{2}+a_{-h} u \frac{2}{x}\right)+k\left(a_{+k} u_{y}^{2}+a_{-k} u \frac{2}{y}\right)\right] \\
\leqq C_{3}\left[\sum_{\Omega_{h}} S_{h} u(0)^{2}+\sum_{s=1}^{p} \Delta t\left(\sum_{\Gamma_{h}}+\sum_{\Gamma_{h}^{\prime}}\right) \bar{g}^{2} \Delta \Gamma+\sum_{s=1}^{p} \Delta t \sum_{\Omega_{h}} S f^{2}\right]
\end{gathered}
$$

where $C_{3}=C_{1} C_{2}+1$. Thus we arrive at

Theorem 3 (Stability). A solution $u$ of the difference problem (3.4), (1.7), (1.10), (1.11) and (3.5) satisfies the energy inequality (3.10) unconditionally for $p \triangle t \leqq T$. (Unconditionally stable)

Theorem 4. (Convergence). Suppose that the boundary $\Gamma$ is sufficiently smooth and a solution $v$ of the differential problem has continuous derivatives $\frac{\partial^{2} v}{\partial t^{2}}, \frac{\partial^{2} v}{\partial x \partial t}, \frac{\partial^{2} v}{\partial y \partial t}, \frac{\partial^{3} v}{\partial x^{3}}, \frac{\partial^{3} v}{\partial x^{2} \partial y}, \frac{\partial^{3} v}{\partial y^{2} \partial x}$ and $\frac{\partial^{3} v}{\partial y^{3}}$ in $\bar{\Omega} \times[0, T]$.

Then the solution $u$ of our difference scheme converges to the corresponding solution $v$ of the differential problem for $\triangle t, h$ and $k \rightarrow 0$ in the sense of

$$
\|v-u\|_{h} \rightarrow 0 \text { uniformly for } p \triangle t \leqq T,
$$

where

$$
\|w\|_{h}=\sum_{\Omega_{h}} S_{h} w(p \triangle t)^{2}+\frac{1}{2} \sum_{s=1}^{p} \triangle t \sum_{\Omega_{h}}\left[h\left(a_{+h} w_{x}^{2}+a_{-h} w_{\bar{x}}^{2}\right)+k\left(a_{+k} w_{y}^{2}+a_{-k} w \frac{2}{y}\right)\right] .
$$

\section{§4. An Elliptic Problem}

Here we consider the Neumann problem for an elliptic equation:

$$
\begin{array}{ll}
-\frac{\partial^{2} u}{\partial x^{2}}-\frac{\partial^{2} u}{\partial y^{2}}+u=f(x, y) & \text { in } \Omega, \\
\frac{\partial u}{\partial n}=g(x, y) & \text { on } \Gamma .
\end{array}
$$

We know that the above problem has one and only one solution under appropriate smoothness condition on $\Gamma, f$ and $g$. Now we can construct a scheme analogous to (1.9) or (3.4) on our net in $\$ 1$ as follows: 


$$
-a_{+h} u_{x}+a_{-h} u_{\bar{x}}-a_{+k} u_{y}+a_{-k} u_{\bar{y}}-\delta_{h} \bar{g}+S_{h} u=S_{h} \bar{f},
$$

with the same boundary condition as (1.7), (1.10) and (1.11) in $\S 1$.

We will prove that this difference scheme is uniquely solvable and its solution converges to an exact solution of (4.1) and (4.2). For the purpose we multiply $u$ on both sides of (4.6) and sum over $\Omega_{h}$, then we have

$$
\begin{aligned}
& -\sum_{i_{G}(j)+1} a_{-h} u_{\bar{x}} u+\sum_{i_{L}(j)-1} a_{+h} u_{x} u-\sum_{\substack{j_{G}(i)>1 \\
i}} a_{-k} u_{\bar{y}} u+\sum_{j_{L}(\bar{i})-1} a_{i} a_{+k} u_{y} u \\
& +\frac{1}{2}\left(\sum_{\Omega_{h}}+\sum_{\substack{i_{G}(j) \\
j}}\right) h a_{+h} u_{x}^{2}+\frac{1}{2}\left(\sum_{\Omega_{h}}+\sum_{i_{L_{j}^{\prime}(j)}}\right) h a_{-h} u_{\frac{2}{x}}^{2} \\
& +\frac{1}{2}\left(\sum_{\Omega_{h}}+\sum_{j_{G}(i)}\right) k a_{+k} u_{y}^{2}+\frac{1}{2}\left(\sum_{\Omega_{h}}+\sum_{j_{L_{i}^{\prime}}(i)}\right) k a_{-k} u \frac{2}{y} \\
& -\sum_{\Gamma_{h}^{\prime}} \bar{g} u \triangle \Gamma+\sum_{\Omega_{h}} S_{h} u^{2}=\sum_{\Omega_{h}} S \bar{f} u,
\end{aligned}
$$

(see the case of a heat equation in §3). Applying the boundary conditions (1.7), (1.10) and (1.11), we have

$$
\begin{aligned}
& \frac{1}{2}\left(\sum_{\Omega_{h}}+\sum_{i_{G_{j}^{(j)}}}\right) h a_{+h} u_{x}^{2}+\frac{1}{2}\left(\sum_{\Omega_{h}}+\sum_{i_{L_{j}(j)}}\right) h a_{-h} u_{\bar{x}}^{2} \\
+ & \frac{1}{2}\left(\sum_{\Omega_{h}}+\sum_{j_{G_{i}(i)}}\right) k a_{+k} u_{y}^{2}+\frac{1}{2}\left(\sum_{\Omega_{h}}+\sum_{j_{L_{i}(i)}}\right) k a_{-k} u \frac{2}{y} \\
+ & \sum_{\Omega_{h}} S_{h} u^{2}=\left(\sum_{\Gamma_{h}}+\sum_{\Gamma_{h}^{\prime}}\right) \triangle \Gamma \bar{g} u+\sum_{\Omega_{h}} S_{h} \bar{f} u .
\end{aligned}
$$

We can estimate the right side by using the Schwarz's inequality and Lemma 1 as follows:

$$
\begin{aligned}
& \frac{1}{2}\left(\sum_{\Omega_{h}}+\sum_{\substack{i_{j}(j) \\
j}}\right) h a_{+h} u_{x}^{2}+\frac{1}{2}\left(\sum_{\Omega_{h}}+\sum_{\substack{i_{L_{j}(j)} \\
j}}\right) h a_{-h} u_{\bar{x}}^{2} \\
+ & \frac{1}{2}\left(\sum_{\Omega_{h}}+\sum_{\substack{j_{i}(i) \\
i}}\right) k a_{+k} u_{y}^{2}+\frac{1}{2}\left(\sum_{\Omega_{h}}+\sum_{\substack{i_{L}(i) \\
j}}\right) k a_{-k} u \frac{2}{y}+\sum_{\Omega_{h}} S_{h} u^{2}
\end{aligned}
$$




$$
\begin{aligned}
& \leqq \varepsilon_{1}\left(\sum_{\Gamma_{h}}+\sum_{\Gamma_{h}^{\prime}}\right) u^{2} \Delta \Gamma+\frac{1}{4 \varepsilon_{1}}\left(\sum_{\Gamma_{h}}+\sum_{\Gamma_{h}^{\prime}}\right) \bar{g}^{2} \Delta \Gamma \\
& +\varepsilon \sum_{\Omega_{h}} S_{h} u^{2}+\frac{1}{4 \varepsilon} \sum_{\Omega_{h}} S_{h} \bar{f}^{2} \\
& \leqq \varepsilon \varepsilon_{1} \sum_{\Omega_{h}}\left[h\left(a_{+h} u_{x}^{2}+a_{-h} u \frac{2}{x}\right)+k\left(a_{+k} u_{y}^{2}+a_{-k} u \frac{2}{y}\right)\right] \\
& +\varepsilon_{1}(C(\varepsilon)+1) \sum_{\Omega_{h}} S_{h} u^{2}+\frac{1}{4 \varepsilon_{1}}\left(\sum_{\Gamma_{h}}+\sum_{\Gamma_{h}^{\prime}}\right) \bar{g}^{2} \triangle \Gamma+\frac{1}{4 \varepsilon_{1}} \sum_{\Omega_{h}} S_{h} \bar{f}^{2},
\end{aligned}
$$

where $\varepsilon_{1}$ is an arbitrarily small constant, $\varepsilon$ and $C^{\prime}(\varepsilon)$ are two constants appearing in Lemma 1 . We take $\varepsilon_{1}$ so small that $\varepsilon \varepsilon_{1}<\frac{1}{4}$ and $\varepsilon_{1}(C(\varepsilon)+1)$ $<\frac{3}{4}$ hold, then we have from the last inequality,

$$
\begin{gathered}
\sum_{\Omega_{h}}\left[h\left(a_{+h} u_{x}^{2}+a_{-h} u_{\bar{x}}^{2}\right)+k\left(a_{+k} u_{y}^{2}+a_{-k} u \frac{2}{y}\right)\right]+\sum_{\Omega_{h}} S_{h} u^{2} \\
\leqq \frac{1}{\varepsilon_{1}}\left(\sum_{\Gamma_{h}}+\sum_{\Gamma_{h}^{\prime}}\right) \bar{g}^{2} \Delta \Gamma+\frac{1}{\varepsilon_{1}} \sum_{\Omega_{h}} S_{h} \bar{f}^{2} .
\end{gathered}
$$

Immediately from the last inequality the uniqueness of the solution of our scheme, and consequently the existence hold. And further we can prove a convergence theorem in the same way as in $\S 2$ and $\S 3$. Thus

Theorem 5 (Convergence). Suppose that the boundary $\Gamma$ of $\Omega$ is sufficiently smooth and a solution of the problem (4.1) and (4.2) has regularity such that third derivatives are continuous in $\bar{\Omega}$. When $h$ and $k$ tend to zero, a solution of our difference problem converges to the corresponding solution of the differential problem in the sense of

$$
\|w\|_{h}=O(h) \quad \text { as } h \rightarrow 0,
$$

where $w$ is the difference between an exact solution and its approximate solution. And

$$
\|w\|_{h}=\sum_{\Omega_{h}}\left[h\left(a_{+h} u_{x}^{2}+a_{-h} u_{\frac{x}{x}}^{2}\right)+k\left(a_{+k} u_{y}^{2}+a_{-k} u \frac{2}{y}\right)+S_{h} u^{2}\right] .
$$

\section{§5. A Numeroำal Experimemt}

In order to test our scheme, we consider a simple problem. Let $\Omega$ be 
a unit circle and $\Gamma$ its boundary. The problem is to find the function $v(x, y)$ satisfying the equations

$$
\begin{array}{lr}
\frac{\partial^{2} v}{\partial t^{2}}-\left(\frac{\partial^{2} v}{\partial x^{2}}+\frac{\partial^{2} v}{\partial y^{2}}\right)=0 & \text { in } \Omega \times[0, T] \\
\frac{\partial v}{\partial n}=2 & \text { on } \Gamma \times[0, T], \\
v(0, x, y)=\frac{\partial v}{\partial t}(0, x, y)=x^{2}+y^{2} & \text { in } \bar{\Omega} .
\end{array}
$$

We can easily see that the exact solution takes the form

$$
v(t, x, y)=2 t^{2}+x^{2}+y^{2}
$$

For simplicity we take the mesh width $h=k=0.1$ and the time step $t=0.05$ which are considerably coarse. For the calculation we have only to consider an octanant of the unit circle because of symmetry (see Fig. 2). 


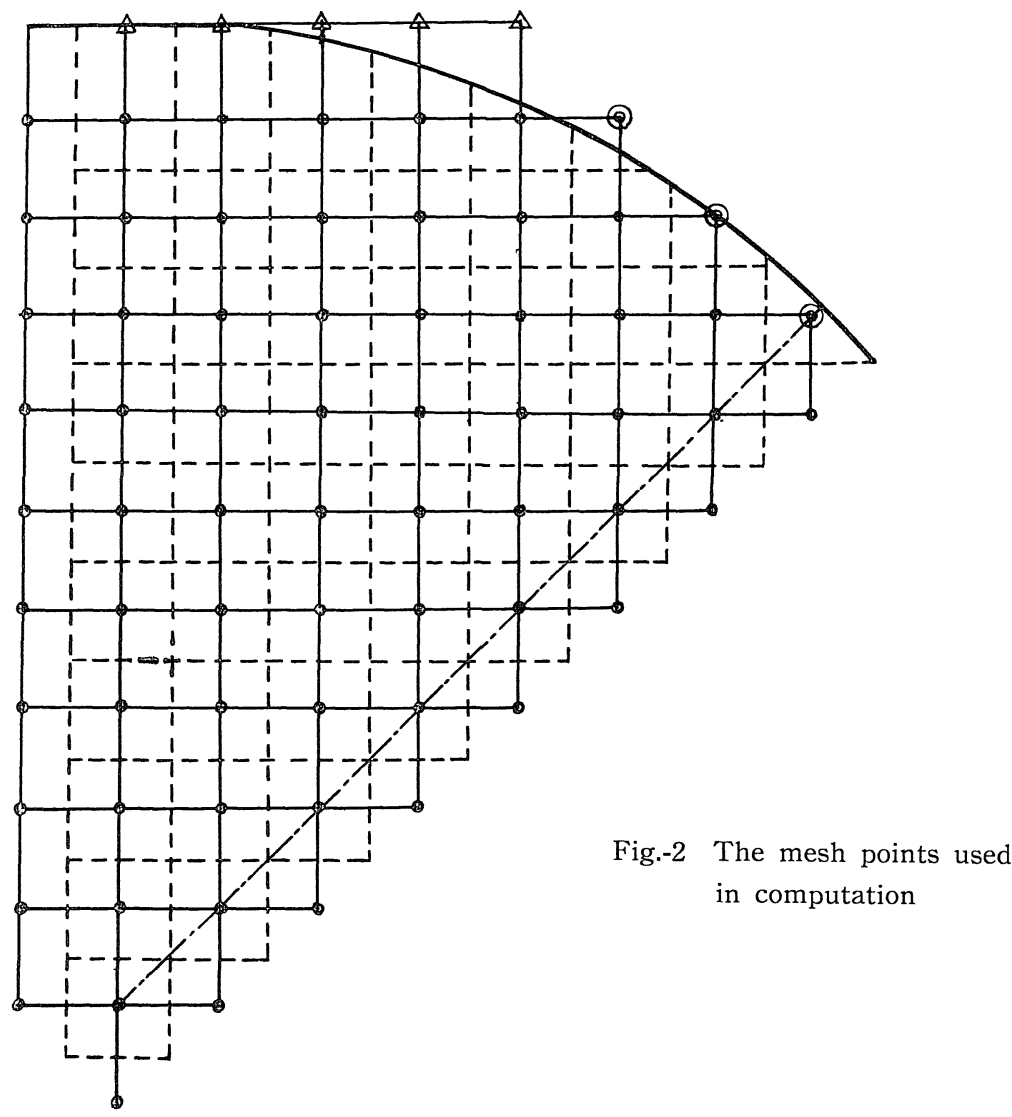

The result of the numerical performance shows stable features of our scheme. We compare the exact solution and our numerical result at $t=10 \triangle t=0.5$ and $t=20 \triangle t=1.0$ (see Table-1 and-2).

We see that at $t=0.5(t=1.0)$ the maximum error which arises near to the boundary is $0.12(0.27)$, while the minimum error is $0.05(0.10)$, which takes place away from the boundary. These results show that the total error has order of $O(h)$ in $0 \leqslant t \leqslant 1.0$. It is the expected matter from our Theorem 2. 


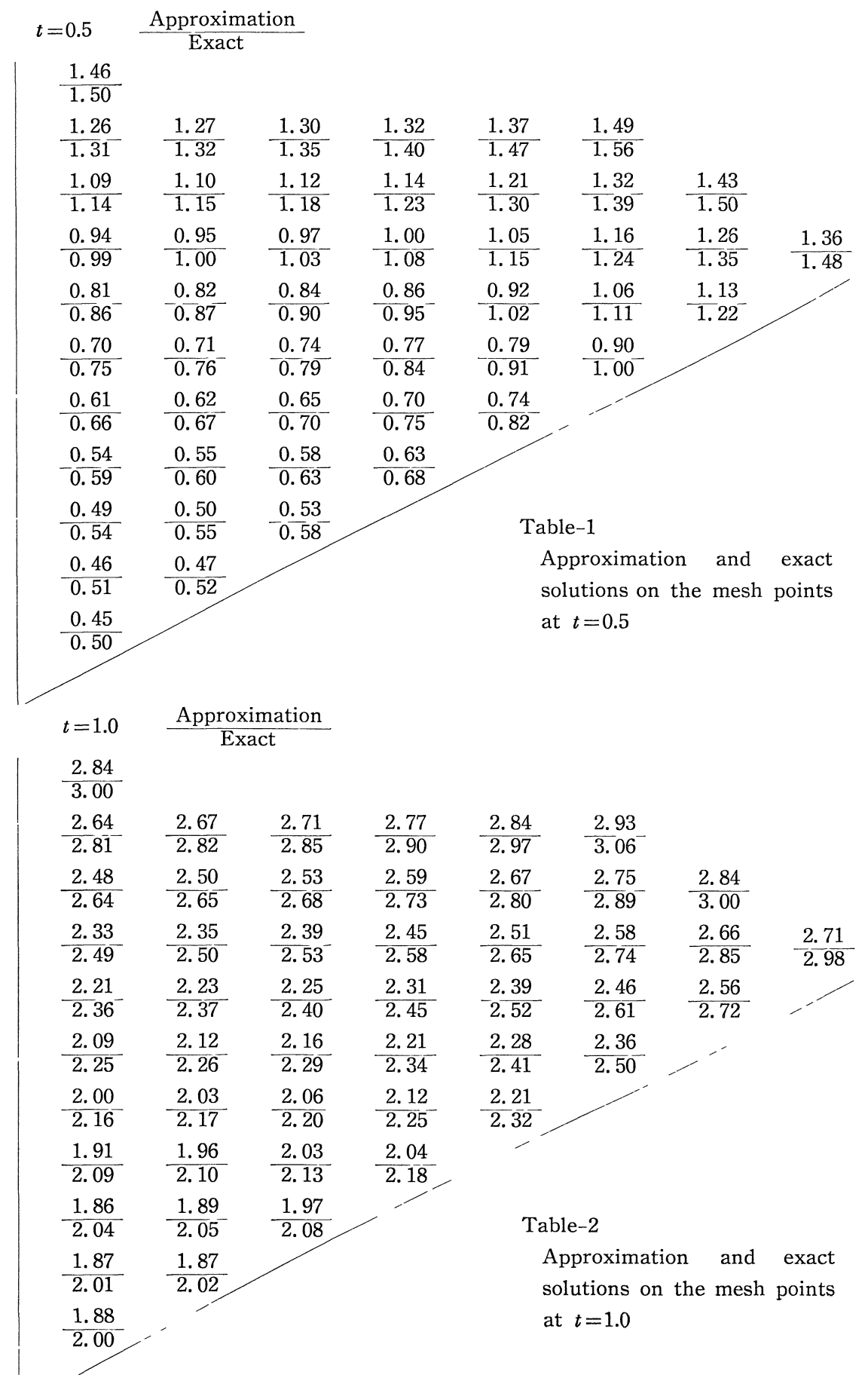




\section{Ackmowledgement}

I should like to thank Prof. M. Yamaguti, Prof. K. Okugawa, Prof. M. Ikeda, Prof. Y. Ohya and Mr. T. Miyoshi for their encouragement and most careful reading of the author's manuscript. I am also indebted to Mrs. S. Naruki for correcting the galley proofs.

\section{References}

[1] Batschelet, E., Über die numerische Aufösung von Randwertproblemen bei elliptischen partiellen Differentialgleichungen, Z. Angew. Math. Physik 3 (1952), 165-193.

[2] Giese, J.H., On the truncation error in a numerical solution of the Neumann problem for a rectangular, J. Math. Phys. 37 (1958), 169-177.

[3] Lebedev, V.I., On the finite difference analogue of the Neumann problem, Dokl. A. N. SSSR 126 (3) (1959), 94-97. (Russian)

[4] The net method for the second kind of boundary value problem of the Poisson's equation, Dokl. A.N. SSSR 127 (4) (1959), 742-745. (Russian)

[5] On the error estimate of the net method for Dirichlet's and Neumann's problems, Dokl. A.N. SSSR 128 (4) (1959), 665-667. (Russian)

[6] Evaluation of the error involved in the method of nets for Neumann's two-dimensional problem, Soviet Math. Dokl. 1 (1960), 703-705.

[7] On the method of nets for the third boundary value problem, Soviet Math. Dokl. 1 (1960), 1056-1060.

[8] Dirichlet's and Neumann's problems on triangular and hexagonal lattices, Soviet Math. Dokl. 2 (1961) 519-522.

[9] Volkov, E.A., On the method of nets for a boundary problem with an oblique and a normal derivative, USSR Comp. Math. and Math. Phys. 1 (1962), 705-722.

[10] - Effective estimates of the error in solution by the method of nets of boundary problems for the Laplace and Poisson equations on a rectangular and a certain triangles, Proc. Steklov Inst. Math. 74 (1966), 57-90.

[11] Wigley, N.M., On the convergence of discrete approximations to solutions of mixed boundary value problems, J. SIAM Numer. Anal. 3 (3) (1966), 372-382.

[12] Demyanovichi, J.K., The net method for some problems in mathematical physics, Soviet Math. Dokl. 5 (1964), 1452-1456.

[13] Friedrichs, K.O., and H.B. Keller, A finite difference scheme for generalized Neumann problems, Numerical Solution of Partial Differential Equations, Academic Press, New York, 1966, pp. 21-34.

[14] Oganesjan, L.A., Convergence of variational difference schemes under improved approximations to the boundary, Soviet Math. Dokl. 7 (1966), 1146-1150.

[15] - Convergence of difference schemes with improved boundary approximation, USSR Comput. Math. and Math. Phys. 5 (1966), 116-134.

[16] Oganesjan L.A., and L.A. Rukhovets, Variational-difference schemes for linear second-order elliptic equations in a two-dimensional region with piecewise smooth boundary, USSR Comput. Math. and Math. Phys. 7 (1968), 129-152.

[17] Oganesjan L.A., and L.A. Rukhovets, Investigation on rate of convergence of variational-difference schemes for second-order elliptic equations in a two- 
dimensional region with smooth boundary, Zh. Vychisl. Mat. i Mat. Fiz. 9 (1969), 1102-1120. (Russian)

[18] Zlamál, M., On the finite element method, Numer. Math. 12 (1968), 394-409.

[19] - On some finite element procedures for solving second order boundary value problems, Numer. Math. 14. (1969), 42-48.

[20] Lions, L., Sur l'approximation des solutions de certain problèmes aux limites, Rend. Sem. Mat. Univ. Padova 32 (1962), 3-54.

[21] Chekhlov, V.I., A boundary value problem for a hyperbolic equation with discontinuous boundary conditions, USSR Comput. Math. and Math. Phys. 5 (1966), 49-62.

[22] Ladyzhenskaya, O.A., A mixed problem for a hyperbolic equation, Gostehizdat, Moscow, 1953 (Russian).

[23] Ikawa, M., Mixed problems for hyperbolic equations of second order, J. Math. Soc. Japan 20 (1968), 580-608. 
